\title{
DEATHSTAR: Nearby AGB stars with the Atacama Compact Array
}

\section{CO envelope sizes and asymmetries: A new hope for accurate mass-loss-rate estimates ${ }^{\star}$}

\author{
S. Ramstedt ${ }^{1}$, W. H. T. Vlemmings ${ }^{2}$, L. Doan ${ }^{1}$, T. Danilovich ${ }^{3}$, M. Lindqvist ${ }^{2}$, M. Saberi ${ }^{2}$, H. Olofsson ${ }^{2}$, \\ E. De Beck ${ }^{2}$, M. A. T. Groenewegen ${ }^{4}$, S. Höfner ${ }^{1}$, J. H. Kastner ${ }^{5}$, F. Kerschbaum ${ }^{6}$, T. Khouri ${ }^{2}$, M. Maercker ${ }^{2}$, \\ R. Montez ${ }^{7}$, G. Quintana-Lacaci ${ }^{8}$, R. Sahai ${ }^{9}$, D. Tafoya ${ }^{2}$, and A. Zijlstra ${ }^{10}$ \\ 1 Theoretical Astrophysics, Division for Astronomy and Space Physics, Department of Physics and Astronomy, Uppsala University, \\ Box 516, 75120 Uppsala, Sweden \\ e-mail: sofia.ramstedt@physics.uu.se \\ 2 Department of Space, Earth and Environment, Chalmers University of Technology, Onsala Space Observatory, \\ 43992 Onsala, Sweden \\ 3 Department of Physics and Astronomy, Institute of Astronomy, KU Leuven, Celestijnenlaan 200D, 3001 Leuven, Belgium \\ 4 Koninklijke Sterrenwacht van België, Ringlaan 3, 1180 Brussels, Belgium \\ 5 Rochester Institute of Technology, Rochester, NY, USA \\ ${ }^{6}$ Department of Astrophysics, University of Vienna, Türkenschanzstr. 17, 1180 Vienna, Austria \\ 7 Smithsonian Astrophysical Observatory, 60 Garden Street, Cambridge, MA 02138, USA \\ 8 Instituto de Física Fundamental (IFF-CSIC), Serrano 123, Madrid, CP 28006, Spain \\ 9 Jet Propulsion Laboratory, MS 183-900, California Institute of Technology, Pasadena, CA 91109, USA \\ 10 Jodrell Bank Centre for Astrophysics, Alan Turing Building, University of Manchester, Manchester M13 9PL, UK
}

Received 8 October 2019 / Accepted 22 April 2020

\begin{abstract}
Context. This is the first publication from the DEATHSTAR project. The overall goal of the project is to reduce the uncertainties of the observational estimates of mass-loss rates from evolved stars on the Asymptotic Giant Branch (AGB).

Aim. The aim in this first publication is to constrain the sizes of the ${ }^{12} \mathrm{CO}$ emitting region from the circumstellar envelopes around 42 mostly southern AGB stars, of which 21 are M-type and 21 are C-type, using the Atacama Compact Array (ACA) at the Atacama Large Millimeter/submillimeter Array. The symmetry of the outflows is also investigated.

Methods. Line emission from ${ }^{12} \mathrm{CO} J=2 \rightarrow 1$ and $3 \rightarrow 2$ from all of the sources were mapped using the ACA. In this initial analysis, the emission distribution was fit to a Gaussian distribution in the $u v$-plane. A detailed radiative transfer analysis will be presented in a future publication. The major and minor axis of the best-fit Gaussian at the line center velocity of the ${ }^{12} \mathrm{CO} J=2 \rightarrow 1$ emission gives a first indication of the size of the emitting region. Furthermore, the fitting results, such as the Gaussian major and minor axis, center position, and the goodness of fit across both lines, constrain the symmetry of the emission distribution. For a subsample of sources, the measured emission distribution is compared to predictions from previous best-fit radiative transfer modeling results.

Results. We find that the $\mathrm{CO}$ envelope sizes are, in general, larger for C-type than for M-type AGB stars, which is as expected if the $\mathrm{CO} / \mathrm{H}_{2}$ ratio is larger in C-type stars. Furthermore, the measurements show a relation between the measured (Gaussian) ${ }^{12} \mathrm{CO}$ $J=2 \rightarrow 1$ size and circumstellar density that, while in broad agreement with photodissociation calculations, reveals large scatter and some systematic differences between the different stellar types. For lower mass-loss-rate irregular and semi-regular variables of both M- and C-type AGB stars, the ${ }^{12} \mathrm{CO} J=2 \rightarrow 1$ size appears to be independent of the ratio of the mass-loss rate to outflow velocity, which is a measure of circumstellar density. For the higher mass-loss-rate Mira stars, the ${ }^{12} \mathrm{CO} J=2 \rightarrow 1$ size clearly increases with circumstellar density, with larger sizes for the higher CO-abundance C-type stars. The M-type stars appear to be consistently smaller than predicted from photodissociation theory. The majority of the sources have $\mathrm{CO}$ envelope sizes that are consistent with a spherically symmetric, smooth outflow, at least on larger scales. For about a third of the sources, indications of strong asymmetries are detected. This is consistent with what was found in previous interferometric investigations of northern sources. Smaller scale asymmetries are found in a larger fraction of sources.

Conclusions. These results for $\mathrm{CO}$ envelope radii and shapes can be used to constrain detailed radiative transfer modeling of the same stars so as to determine mass-loss rates that are independent of photodissociation models. For a large fraction of the sources, observations at higher spatial resolution will be necessary to deduce the nature and origin of the complex circumstellar dynamics revealed by our ACA observations.
\end{abstract}

Key words. stars: AGB and post-AGB - stars: mass-loss - stars: winds, outflows - circumstellar matter

\footnotetext{
* The reduced datacubes are only available at the CDS via anonymous ftp to cdsarc.u-strasbg.fr (130.79.128.5) or via http://cdsarc.u-strasbg.fr/viz-bin/cat/J/A+A/640/A133
} 


\section{Introduction}

Stars with zero-age-main-sequence masses in the range of $\sim 0.8-8 M_{\odot}$ evolve into asymptotic giant branch (AGB) stars during the late stages of their evolution. The heavy mass loss during the AGB makes the stars major contributors of newly synthesized elements and dust to their surroundings. Understanding the mass-loss process is crucial for comprehending the evolution of stars in this mass range, but also for extragalactic population studies. Mass-loss rates, $\dot{M}$, on the AGB are found to range from $\sim 10^{-8}-10^{-4} M_{\odot} \mathrm{yr}^{-1}$ (e.g., Höfner \& Olofsson 2018, and references therein). It is challenging to find reliable observational methods to measure mass-loss rates covering this wide range (Ramstedt et al. 2008), but it is crucial since the measurements will provide key constraints for theoretical models (e.g., Eriksson et al. 2014; Marigo et al. 2016; Bladh et al. 2019). Wind formation is studied using dynamical wind models (e.g., Höfner 2008; Eriksson et al. 2014; Bladh et al. 2015; Höfner et al. 2016) with the ultimate goal of developing a predictive theory of AGB mass loss. This will permit reliable estimates of dust production and, for example, the intrinsic reddening of distant galaxies (e.g., Conroy 2013). These models are constrained using observed wind properties, that is, $\dot{M}$ and wind velocities, $v_{\infty}$. However, with the derived $\dot{M}$ uncertainties reaching as high as a factor of three (within the adopted spherically symmetric model, Ramstedt et al. 2008), the dynamical wind models cannot be sufficiently well constrained (e.g., Fig. 7 in the recent paper by Bladh et al. 2019 shows how the wind parameters vary with model input parameters).

Observations of ${ }^{12} \mathrm{CO}$ radio line emission (originating in the circumstellar envelope, CSE, which is created by the wind), in combination with detailed radiative transfer, is considered to be the most reliable method for determining AGB wind properties (e.g., Höfner \& Olofsson 2018, and references therein). The poorly constrained size of the ${ }^{12} \mathrm{CO}$ envelope is a remaining, significant source of uncertainty for the mass-loss rates estimated using this method. The generally adopted strategy is to use an envelope-sized estimate based on the photodissociation model by Mamon et al. (1988). Major uncertainties are that this model assumed a standard interstellar radiation field (Draine 1978), and that numerical methods and shielding functions have been updated since then (e.g., Groenewegen 2017; Saberi et al. 2019). In radiative transfer models that are intended to determine wind parameters from ${ }^{12} \mathrm{CO}$ line observations, the ${ }^{12} \mathrm{CO}$ envelope size is estimated using a functional fit to the Mamon et al. (1988) photodissociation model results. The envelope size is a function of the wind parameters, including the ${ }^{12} \mathrm{CO}$ abundance, which increase with density, that is, $\dot{M} / v_{\mathrm{e}}$. A more exact way to determine the ${ }^{12} \mathrm{CO}$ envelope size (e.g., independent of our knowledge of shielding functions) is to constrain it directly using interferometry. Direct observations will further improve the accuracy, since the radiation environment changes from source to source .

A pioneering interferometric survey of AGB (and post-AGB) CO CSEs was performed by Neri et al. (1998). Forty-six sources were mapped in ${ }^{12} \mathrm{CO} J=1 \rightarrow 0$ (from now on $\mathrm{CO}(1-0)$, etc) emission with three antennas on the Plateau de Bure interferometer combined with the IRAM $30 \mathrm{~m}$ telescope. The sample was selected on evolutionary status, declination, IR color, and CO line strength. They found a good agreement between measured $\mathrm{CO}(1-0)$ sizes and photodissociation radii based on the model by Mamon et al. (1988); however, as expected, they observed significant scatter. They also concluded that about $30 \%$ of the envelopes show significant asymmetry. This investigation was later followed up with considerably improved capabilities at the
Plateau de Bure by the COSAS program (Castro-Carrizo et al. 2010). The goal was to investigate the morphologies of the envelopes, in particular during the transition to post-AGB, and this sample contained several sources at later evolutionary stages than the AGB. Castro-Carrizo et al. (2010) presented detailed maps of the $\mathrm{CO}(1-0)$ and $(2-1)$ emission from 16 sources and thoroughly discussed each source. The synthesized beams were typically of the order $3-5^{\prime \prime}$ and $1-2^{\prime \prime}$ for $\mathrm{CO}(1-0)$ and $(2-1)$, respectively. They found that the measured envelope sizes were, on average, slightly larger than expected from the photodissociation model by Mamon et al. (1988). They further concluded that the AGB envelopes generally show round shapes and approximately isotropic expansion, while most later sources, that is postAGB, exhibit axial symmetry and fast bipolar flows.

We have started a new project called DEATHSTAR ${ }^{1}$ (DEtermining Accurate mass-loss rates for THermally pulsing AGB STARs) in which the overall aim is to better constrain the measurements of AGB mass-loss rates. Observations of a large sample of "typical" AGB envelopes (sources with known strong deviations from spherical symmetry have been omitted), covering the full range of AGB stellar and wind parameters, will be analyzed in detail in future work. The already-available data base of CO lower- $J$ transitions will be modeled, together with available and new interferometric observations using updated radiative transfer models. In this first paper, new interferometric data of $\mathrm{CO}(2-1)$ and (3-2) emission obtained with the Atacama Compact Array (ACA) at the Atacama Large Millimeter/submillimeter Array (ALMA) are presented. The sample selection is explained in Sect. 2. The observations, data reduction, and data analysis are outlined in Sect. 3. The results with an analysis of the CO line emission distributions (size and morphology) and an overview of the detections of emission from other molecular species are presented in Sect. 4. Finally, the results are discussed and summarized in Sects. 5 and 6.

\section{The sample}

The full sample for which the circumstellar CO line emission will be modeled consists of the $\sim 180 \mathrm{C}-, \mathrm{M}-$, and S-type AGB stars analyzed in Schöier \& Olofsson (2001), González Delgado et al. (2003), and Ramstedt et al. (2006) together with additional sources presented in Danilovich et al. (2015). In this initial paper, the new interferometric data for the southern M- and C-type stars are presented. Some of the available sample statistics for the full $\sim 180$ star DEATHSTAR sample are shown in Fig. 1. The distance distribution (Fig. 1, middle) is compared with the estimated distribution in the solar neighborhood (Jura 1990; Jura \& Kleinmann 1992; Jura et al. 1993). The estimated distribution is derived from 2MASS and ground-based observations (Jura \& Kleinmann 1990) and assumes a smooth distribution of 40 C-type stars $\mathrm{kpc}^{-2}$, a scale height of $200 \mathrm{pc}$, and that there are a third as many S-type as C-type stars. For the full $\sim 180$ star DEATHSTAR sample, the C-type stars from Schöier \& Olofsson (2001) are all brighter than $K=2 \mathrm{mag}$. The M-type sample consists of the non-Mira stars from the General Catalog of Variable Stars (GCVS; Samus' et al. 2017) with quality flag 3 in the IRAS 12,25 , and $60 \mu \mathrm{m}$ bands and $60 \mu \mathrm{m}$ flux $\gtrsim 3 \mathrm{Jy}$ with the addition of the Mira stars in González Delgado et al. (2003). The S-type sample also consists of stars that have good quality flux measurements in the IRAS 12,25 , and $60 \mu \mathrm{m}$ bands, that are found in the General Catalog of Galactic S stars, and that are detected in Tc

\footnotetext{
wWw. astro.uu.se/deathstar
} 

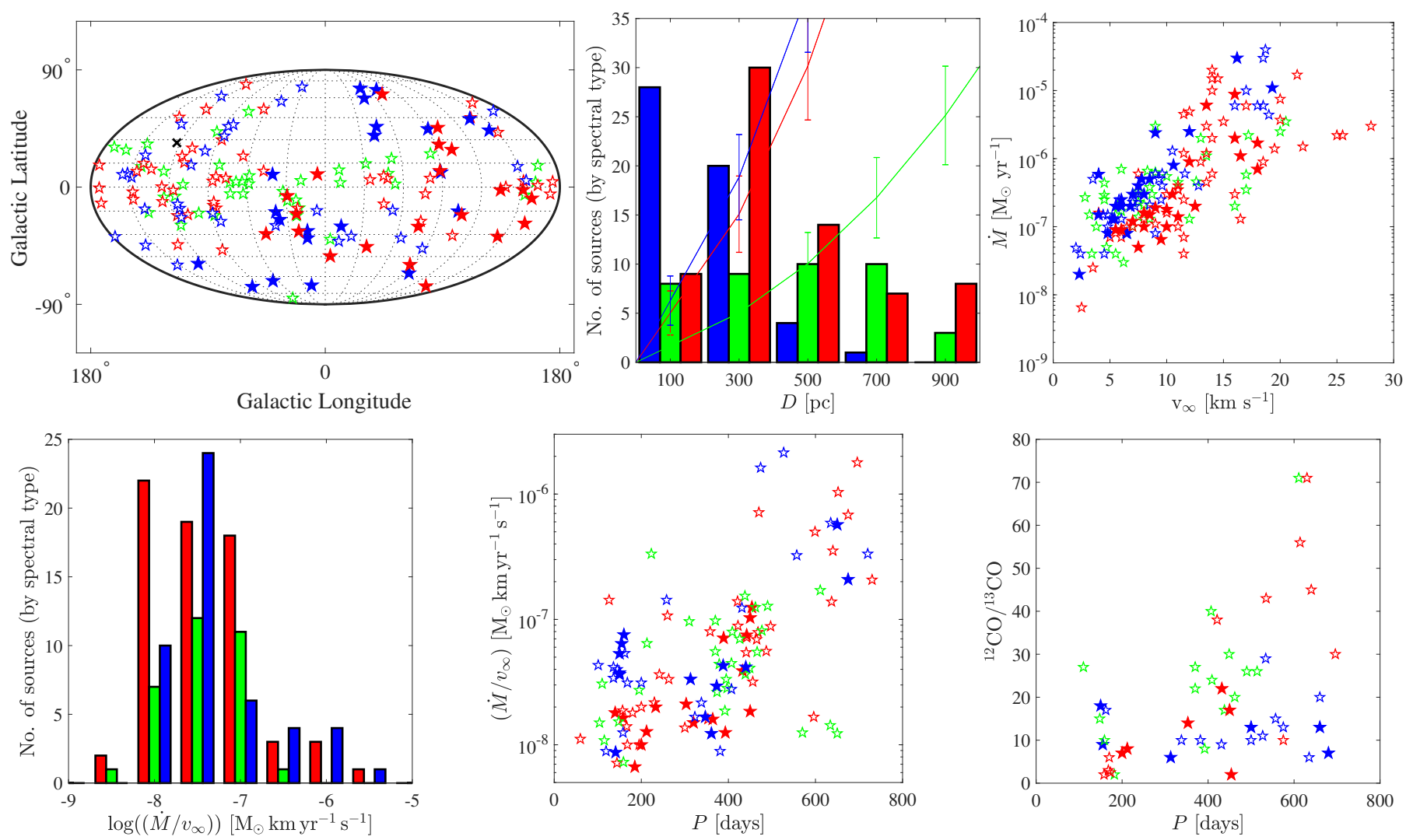

Fig. 1. Sample statistics of the full $\sim 180$ star DEATHSTAR sample. M-type stars are blue, C-type stars are red, and S-type stars are green. Filled symbols mark the stars included in this paper. Upper left: galactic distribution of the full sample. Upper middle: distance, $D$, distribution. These are preliminary distances from previous publications (see text and Table 1). The solid lines show the expected distributions for each spectral type with Poisson errors (Jura 1990; Jura \& Kleinmann 1992; Jura et al. 1993). Upper right: wind properties (mass-loss rate, $\dot{M}$, and terminal expansion velocity, $v_{\infty}$ ) from previous publications. See text for references. Lower left: $\dot{M} / v_{\infty}$ distribution for the full sample. We note that $\dot{M} / v_{\infty}$ is a proxy for the wind density. Lower middle: $\dot{M} / v_{\infty}$ as a function of the pulsational period. Lower left: ${ }^{12} \mathrm{CO} /{ }^{13} \mathrm{CO}$ ratio for the sample sources (Ramstedt \& Olofsson 2014) is plotted against the pulsational period. Both parameters are expected to increase as the stars evolve. See text for a further discussion.

and are hence intrinsic. The completeness of the S-type sample is discussed in Ramstedt et al. (2009) and it is thought to be complete out to $600 \mathrm{pc}$. Furthermore, stars of all three spectral types are only included in the sample if they are detected in $\mathrm{CO}$ radio line emission, which could be reproduced under the assumption of spherical symmetry. Sources that show strongly asymmetric line profiles when observed with single-dish telescopes, or with known CO-detached shells, are hence not included (e.g., R Scl, U Ant, EP Aqr, and $\pi^{1}$ Gru). In this paper, we also exclude stars that have previously been observed with ALMA; however, they will be included in the future analysis. The lower panel of Fig. 1 shows that the stellar and wind parameters of the full $\sim 180$ star sample cover the ranges expected for AGB stars. As expected, fewer stars are found at the high end. The sample is biased to mass-losing stars since only stars that are previously detected in $\mathrm{CO}$ radio line emission are included. It is also likely that the full range of AGB masses is not covered simply because higher mass stars are rare. It is our assessment that the full $\sim 180$ star DEATHSTAR sample is representative of Galactic mass-losing AGB stars and covers the relevant ranges of wind and stellar parameters to provide the necessary constraints for theoretical models.

The 42 stars (21 M-type, 21 C-type), which were observed with ALMA ACA in Cycle 4 and for which the data are presented in this paper, are listed in Table 1 with the variability type (as listed in the GCVS), mass-loss rate, final wind velocity, dis- tance according to the previous analysis in Schöier \& Olofsson (2001), González Delgado et al. (2003), and Danilovich et al. (2015), and Gaia data release 2 (DR2) (Gaia Collaboration 2016, 2018) distance from Bailer-Jones et al. (2018). The mass-loss rates and wind velocities were estimated by reproducing several low- $J$ CO lines for each source using the non-LTE, nonlocal, spherically symmetric radiative transfer code that was first presented in Schöier \& Olofsson (2001). The $\mathrm{CO} / \mathrm{H}_{2}$ abundance ratio, which is necessary to derive the total gas mass-loss rate, is assumed to be $2 \times 10^{-4}$ for the M-type stars and $1 \times 10^{-3}$ for the C-type stars. The results from the photodissociation model by Mamon et al. (1988) was used in the radiative transfer modeling. In order to give consistent mass-loss rates and distances, the distances adopted here (and listed in Table 1) are the same as those used in the papers listed above. The distances will be updated as part of the future radiative transfer analysis planned for the full sample. For the semi-regular M-type stars, a stellar bolometric luminosity of $4000 L_{\odot}$ was adopted. For M-type Mira variables and some C-type stars, the period-luminosity relations from Whitelock et al. (1994) and Groenewegen \& Whitelock (1996) were used to estimate the luminosity, respectively. From the luminosity, the distances were determined by either using two blackbodies or by using the dust radiative transfer code DUSTY (Ivezic et al. 1999) to model the spectral energy distribution (SED). For the remaining C-type stars, the distance 
Table 1. Forty-two sources observed with the ACA by spectral type and variability type listed in ascending mass-loss-rate order.

\begin{tabular}{|c|c|c|c|c|c|}
\hline Source & $\begin{array}{c}\text { Variability } \\
\text { type }\end{array}$ & $\begin{array}{c}\dot{M}^{(a)} \\
{\left[M_{\odot} \mathrm{yr}^{-1}\right]}\end{array}$ & $\begin{array}{c}v_{\infty}^{(a)} \\
{\left[\mathrm{km} \mathrm{s}^{-1}\right]}\end{array}$ & $\begin{array}{c}D \\
{[\mathrm{pc}]}\end{array}$ & $\begin{array}{r}D_{\text {Gaia }} \\
{[\mathrm{pc}]}\end{array}$ \\
\hline \multicolumn{6}{|c|}{ M-type semi-regular and irregular stars: } \\
\hline $\mathrm{L}_{2}$ Pup & $\mathrm{SRb}$ & $2 \times 10^{-8}$ & 2.3 & 85 & 140 \\
\hline W Hya & $\mathrm{SRa}$ & $8 \times 10^{-8}$ & 6.5 & 65 & 160 \\
\hline T Mic & $\mathrm{SRb}$ & $8 \times 10^{-8}$ & 4.8 & 130 & 190 \\
\hline Y Scl & $\mathrm{SRb}$ & $1.3 \times 10^{-7}$ & 5.2 & 330 & 310 \\
\hline V1943 Sgr & $\mathrm{Lb}$ & $1.3 \times 10^{-7}$ & 5.4 & 150 & 665 \\
\hline BK Vir & $\mathrm{SRb}$ & $1.5 \times 10^{-7}$ & 4.0 & 190 & 235 \\
\hline V Tel & $\mathrm{SRb}$ & $2 \times 10^{-7}$ & 6.8 & 290 & 400 \\
\hline SU Vel & $\mathrm{SRb}$ & $2 \times 10^{-7}$ & 5.5 & 250 & 290 \\
\hline UY Cet & $\mathrm{SRb}$ & $2.5 \times 10^{-7}$ & 6.0 & 300 & 320 \\
\hline SV Aqr & $\mathrm{Lb}$ & $3 \times 10^{-7}$ & 8.0 & 470 & 390 \\
\hline SW Vir & $\mathrm{SRb}$ & $4 \times 10^{-7}$ & 7.5 & 120 & 300 \\
\hline $\mathrm{CW}$ Cnc & $\mathrm{Lb}$ & $5 \times 10^{-7}$ & 8.5 & 280 & 210 \\
\hline RT Vir & $\mathrm{SRb}$ & $5 \times 10^{-7}$ & 7.8 & 170 & 490 \\
\hline $\mathrm{R}$ Crt & $\mathrm{SRb}$ & $8 \times 10^{-7}$ & 10.6 & 170 & 240 \\
\hline \multicolumn{6}{|c|}{ M-type Mira stars: } \\
\hline R Leo & M & $2 \times 10^{-7}$ & 6.0 & 130 & 70 \\
\hline R Hya & M & $3 \times 10^{-7}$ & 7.0 & 150 & 225 \\
\hline R Hor & M & $5.9 \times 10^{-7}$ & 4.0 & 310 & 310 \\
\hline RR Aql & M & $2.4 \times 10^{-6}$ & 9.0 & 530 & 320 \\
\hline IRC-10529 & M & $2.5 \times 10^{-6}$ & 12.0 & 270 & - \\
\hline WX Psc & M & $1.1 \times 10^{-5}$ & 19.3 & 600 & - \\
\hline IRC +10365 & $\mathrm{M}$ & $3 \times 10^{-5}$ & 16.2 & 750 & 360 \\
\hline \multicolumn{6}{|c|}{$C$-type semi-regular and irregular stars: } \\
\hline TW Oph & $\mathrm{SRb}$ & $5 \times 10^{-8}$ & 7.5 & $280^{(b)}$ & 590 \\
\hline NP Pup & $\mathrm{Lb}$ & $6.5 \times 10^{-8}$ & 9.5 & $420^{(b)}$ & 470 \\
\hline TW Hor & $\mathrm{SRb}$ & $9 \times 10^{-8}$ & 5.5 & $400^{(b)}$ & 420 \\
\hline T Ind & $\mathrm{SRb}$ & $9 \times 10^{-8}$ & 6.0 & $570^{(b)}$ & 610 \\
\hline RT Cap & $\mathrm{SRb}$ & $1 \times 10^{-7}$ & 8.0 & $450^{(c)}$ & 430 \\
\hline AQ Sgr & $\mathrm{SRb}$ & $2.5 \times 10^{-7}$ & 10.0 & $420^{(d)}$ & 560 \\
\hline U Hya & $\mathrm{SRb}$ & $1.2 \times 10^{-7}$ & 7.0 & $160^{(b)}$ & 170 \\
\hline W Ori & $\mathrm{SRb}$ & $1.4 \times 10^{-7}$ & 11.0 & $220^{(b)}$ & 1010 \\
\hline V Aql & $\mathrm{SRb}$ & $1.5 \times 10^{-7}$ & 8.5 & $370^{(b)}$ & 400 \\
\hline Y Pav & $\mathrm{SRb}$ & $1.6 \times 10^{-7}$ & 8.0 & $360^{(c)}$ & - \\
\hline $\mathrm{X}$ Vel & SR & $1.8 \times 10^{-7}$ & 10.0 & $310^{(c)}$ & 640 \\
\hline Y Hya & $\mathrm{SRb}$ & $1.9 \times 10^{-7}$ & 9.0 & $350^{(b)}$ & 475 \\
\hline SS Vir & SRa & $2 \times 10^{-7}$ & 12.5 & $540^{(c)}$ & 670 \\
\hline W CMa & $\mathrm{Lb}$ & $3 \times 10^{-7}$ & 10.5 & $450^{(d)}$ & 555 \\
\hline \multicolumn{6}{|c|}{ C-type Mira stars: } \\
\hline R Lep & M & $7 \times 10^{-7}$ & 18.0 & $250^{(b)}$ & 420 \\
\hline CZ Hya & M & $9 \times 10^{-7}$ & 12.0 & $960^{(c)}$ & 3300 \\
\hline R For & M & $1.1 \times 10^{-6}$ & 16.5 & $610^{(c)}$ & 630 \\
\hline R Vol & M & $1.7 \times 10^{-6}$ & 18.0 & $730^{(c)}$ & 840 \\
\hline RV Aqr & M & $2 \times 10^{-6}$ & 16.0 & $670^{(c)}$ & 860 \\
\hline V688 Mon & M & $6.1 \times 10^{-6}$ & 13.5 & $1770^{(e)}$ & 500 \\
\hline V1259 Ori & M & $8.8 \times 10^{-6}$ & 16.0 & $1600^{(e)}$ & - \\
\hline
\end{tabular}

Notes. The columns give wind properties (mass-loss rate, $\dot{M}$, and final expansion velocity, $v_{\infty}$ ), and preliminary distance, $D$, from previous publications (see text for details). The final column gives the distances from Gaia data release 2 (Bailer-Jones et al. 2018) for comparison purposes (see also Fig. 2). ${ }^{(a)}$ From the previous analysis. See text for references. ${ }^{(b)}$ HIPPARCos parallax. ${ }^{(c)}$ Period-luminosity relation (Groenewegen \& Whitelock 1996). ${ }^{(d)}$ Assuming $4000 L_{\odot} .{ }^{(e)}$ Adopted from Menzies et al. (2006).

was estimated directly from the original HIPPARCOS (ESA 1997) parallax (The HIPPARCOS and Tycho catalogs 1997) or adopted from Menzies et al. (2006). The method used for each C-type star is noted in Table 1. Figure 2 shows the comparison between the adopted distances from a previous analysis and the new Gaia DR2 distances for the full sample (Bailer-Jones et al. 2018).

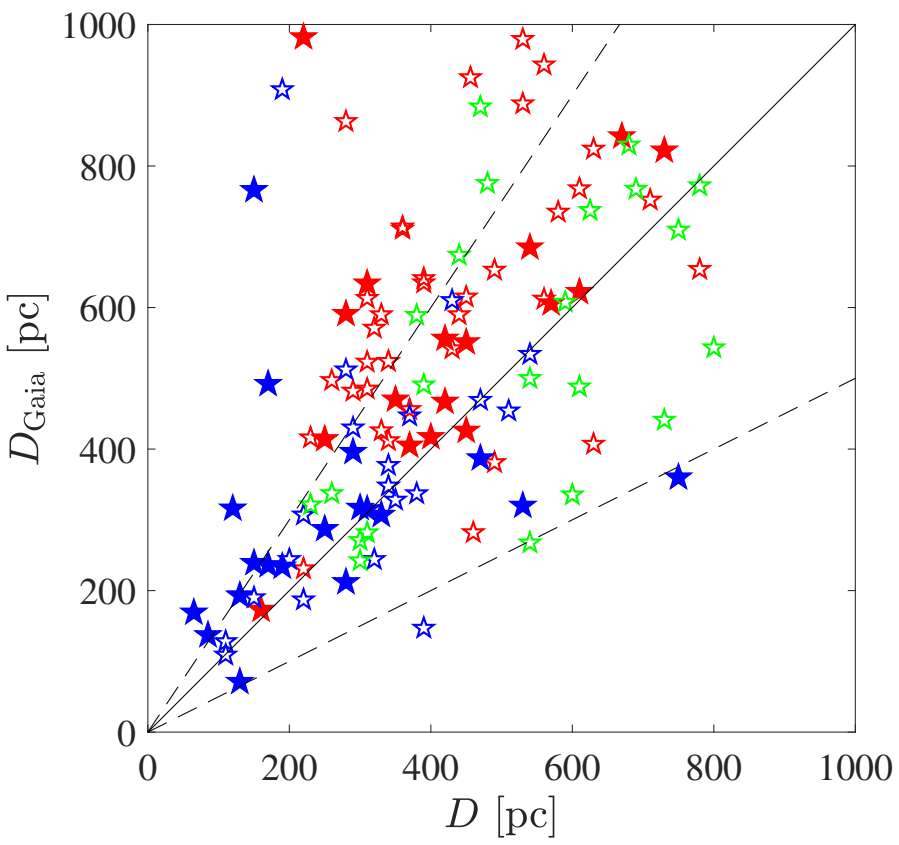

Fig. 2. Comparison between distances from Gaia DR2 parallaxes and the adopted distances from the previous analysis (see text for details) for the full $\sim 180$ star DEATHSTAR sample. Red symbols are carbon stars, blue symbols are M-type stars, and green symbols are S-type stars. Filled symbols are the sources included in this paper. The solid, black line marks a one-to-one correlation, while the dashed lines show the range of $\pm 50 \%$.

The spread is very large and the uncertainties affecting the Gaia DR2 distances for these types of stars are further discussed in Appendix A. The Gaia DR2 distances are typically larger than the adopted distances. The mass-loss rates given in Table 1 scale with distance as $D^{n}$ where $1.4 \lesssim n \lesssim 1.9$ (Ramstedt et al. 2008) and would, in general, be larger than those in Table 1 if Gaia DR2 distances are used in the analysis.

\section{Observations, data reduction, and analysis}

\subsection{Observations with the ACA}

The 42 sample sources listed in Table 1 were observed with the ACA in stand-alone mode in Cycle 4 in Bands 6 and 7 (project codes: 2016.2.00025.S and 2017.1.00595.S). The correlator was set up with four spectral windows in each band. In Band 6 the windows were centered on 216.4, 218.3, 230.7, and 232.1 GHz. In Band 7 they were centered on 330.75, 332.25, 343.52, and $345.6 \mathrm{GHz}$. Line emission from ${ }^{12} \mathrm{CO} J=2 \rightarrow 1,3 \rightarrow 2$, and ${ }^{13} \mathrm{CO} J=3 \rightarrow 2$ were covered in this setup, as well as emission from a large number of other chemically interesting molecules, including $\mathrm{SiO}, \mathrm{SiS}$, and $\mathrm{CS}$, for example. The spectral resolution of the imaged data was set to $0.75 \mathrm{~km} \mathrm{~s}^{-1}$ in the ${ }^{12} \mathrm{CO}$ and ${ }^{13} \mathrm{CO}$ windows and to 1.0 and $1.5 \mathrm{~km} \mathrm{~s}^{-1}$ in the other spectral windows in Band 6 and 7, respectively.

All data were calibrated using the standard pipeline scripts and imaged using the Common Astronomy Software Applications package (CASA; McMullin et al. 2007). Self-calibration was performed using a small number of channels, typically two, across the peak ${ }^{12} \mathrm{CO}$ line emission and applied to all sources in both bands. This improved the signal-to-noise ratio by $10-15 \%$ on average. All data were cleaned initially using 10000 iterations. For sources brighter than $\sim 20 \mathrm{Jy}$, we found that 
further cleaning iterations was necessary to recover the signal and finally 20000 iterations were applied to all bright ( $>20 \mathrm{Jy}$ ) sources. The processed data have improved the quality compared to the data products provided in the ALMA archive. Each spectral window in both bands were imaged separately and can be accessed through $\mathrm{CDS}^{2}$.

The full width at half-maximum (FWHM) beam widths and root mean square (rms) noise levels at a velocity resolution of $0.75 \mathrm{~km} \mathrm{~s}^{-1}$, which were measured in the emission-free channels in the ${ }^{12} \mathrm{CO}$ line window of the final images in both bands, are given in Table B.1 for all sources. The science goal beam widths and rms values at $1.5 \mathrm{~km} \mathrm{~s}^{-1}$ were $5.5^{\prime \prime}$ and $40 \mathrm{mJy}^{\text {beam }}{ }^{-1}$ as well as 3-4" and $50 \mathrm{mJy}^{\prime \prime}$ beam $^{-1}$ in Bands 6 and 7, respectively. For a significant fraction of the data sets, the beams are larger than what was aimed for as well as elongated; this is an effect of the project being partly observed as a filler program with some of the sources at low elevation. The maximum recoverable scale will cover a range depending on the exact antenna configuration and frequency, but on average it was $25^{\prime \prime} \pm 4^{\prime \prime}$ and $18^{\prime \prime} \pm 2^{\prime \prime}$ for Band 6 and 7, respectively. The data quality is sufficient for the DEATHSTAR project goals; however, as is discussed below, there are compelling reasons to follow up on a majority of the sources with higher-spatial-resolution observations.

\subsection{Fitting the emission distribution in the $u v$-plane}

The first step is to fit the visibility data in the $\mathrm{CO}(2-1)$ and (3-2) measurement sets with Gaussian distributions for all sources using the CASA task UVMULTIFIT (Martí-Vidal et al. 2014). The least-square fit gives the center position coordinates, the major axis and the axis ratio of the best-fit Gaussian, and the position angle of the major axis for each channel over which the fit has been performed. This provides initial estimates for the sizes of the emitting regions and indications of deviations from symmetry (as described below). The next step will be to perform detailed radiative transfer modeling that is constrained by the available multitransition single-dish, including the $\mathrm{CO}(1-0)$ line, and interferometry data for each source to determine the size of the $\mathrm{CO}$ envelope and to fit the emission distributions. This will be presented in a future publication.

\subsection{Emission distributions from $1 D$ radiative transfer models}

Emission distributions for the $\mathrm{CO}(2-1)$ and (3-2) lines were calculated from our previous best-fit radiative transfer model results for a subsample of sources. This is a first step toward full radiative transfer modeling in order to reproduce the data available for each source (planned for a future publication). The subsample consists of six stars: three M-type and three C-type stars of which there is one low-, one intermediate-, and one high-massloss-rate source for each type. The models were directly adapted from the previous best-fit models (Danilovich et al. 2015, see also Table 1) and no attempt has been made to improve the fit to the ALMA ACA data. Instead, the purpose is to start evaluating the validity of the photodissociation model used to estimate the size of the circumstellar CO envelopes in the models. For these sources, the original radiative transfer used the photodissociation radius from Mamon et al. (1988). The output from the best-fit radiative transfer models of the six sources was used to create image cubes with an imaging ray-tracing program that is part of the radiative transfer package developed by Schöier (2000). The image cubes were created with $32 \times 32$ pixels with sizes of $0 \prime: 8$ and 0,7 for each channel across the $\operatorname{CO}(2-1)$ and (3-2)

\footnotetext{
2 See also www. astro.uu. se/deathstar
}

line, respectively. Once image cubes were created, they were run through the ALMA simulator in CASA to simulate observations with the same configuration and under the same conditions as during the ACA observations of each respective source.

\section{Results}

\subsection{Line profiles}

The CO $J=2 \rightarrow 1$ and $3 \rightarrow 2$ line profiles were generated from the image cubes across the lines and are presented in Figs. D.1-D.2. A circular aperture of $18^{\prime \prime}$ was used for both transitions because this covered the emitting region without reaching lower-fidelity regions that are close to the image edges. Table B.1 gives the peak flux, the center velocity, and the total velocity width of each line. The peak value was measured at the maximum point across the line. The center velocity was measured at the center between the two points where the flux reaches $5 \%$ of the peak value at the extreme velocities. The total width is the velocity width between the two 5\%-peak-flux-value points. The line profiles show an interesting structure that is likely indicative of circumstellar dynamics and which was revealed due to the very high signal-to-noise ratio attained. For example, some lines are distinctly asymmetric (see e.g., R Hya and SS Vir). Furthermore, some lines seem to show extended wings (see e.g., $\mathrm{L}^{2}$ Pup and TW Hor). Indications of circumstellar inhomogeneities and nonisotropic kinematics are further discussed below (Sect. 4.3).

\subsection{Emission distribution}

\subsubsection{Gaussian distribution fits}

The results of fitting the data using Gaussian visibility distributions are given in Table 2. The second column gives two times the predicted photodissociation radius, $R_{\mathrm{p}}$, over the distance, $D$ (from Table 1), as a measure of the expected size of the $\mathrm{CO}$ line emitting region. The photodissociation radius, which was measured as the radius where the $\mathrm{CO}$ abundance has dropped to half of its initial value, was calculated using Eqs. (10) and (11) from Schöier \& Olofsson (2001), which were derived (Stanek et al. 1995) by fitting the results of the CO photodissociation model by Mamon et al. (1988). The parameter values given in Table 1 were used for the calculation. Table 2 also lists the beam-deconvolved FWHM major axis and the axis ratio of the best-fit Gaussian at the center velocity (from Table B.1). The error is the average error of the fits in the two channels adjacent to the center velocity. When the error is large when the center channels are strongly affected by resolved-out flux, for example (see discussion in Sect. 4.3 and Figs. E.1-E.2), the results are given without errors and marked by a colon (:).

There are good reasons (from previous radiative transfer models, e.g., Ramstedt \& Olofsson 2014) to expect that the $\mathrm{CO}(2-1)$ line will be excited almost throughout the entire CSE. However, without detailed models for individual sources, we cannot estimate how well the fitted Gaussian semi-major axis will correspond to the photodissociation radius, or by what factor it may deviate (see Sect. 4.2.2). In Fig. 3, we illustrate the reliability of using the measured Gaussian size as a proxy for the size of the emitting region and for the photodissociation radius, $R_{\mathrm{p}}$, based on the recent models by Saberi et al. (2019). These models include a more complete treatment of CO selfshielding and yield radii that are mostly similar to Mamon et al. (1988), but they can be up to $\sim 40 \%$ smaller for some combinations of mass-loss rates and initial CO abundances. We used 
Table 2. Results from the Gaussian visibility distribution fitting.

\begin{tabular}{|c|c|c|c|c|c|c|}
\hline \multirow[b]{2}{*}{ Source } & \multirow{2}{*}{$\begin{array}{c}2 R_{\mathrm{p}} \\
D \\
{\left[{ }^{\prime \prime}\right]}\end{array}$} & \multicolumn{2}{|c|}{$\mathrm{CO}(2-1)$} & \multicolumn{2}{|c|}{$\mathrm{CO}(3-2)$} & \multirow{2}{*}{$\begin{array}{c}\text { Asym. } \\
\text { Feat. }\end{array}$} \\
\hline & & $\begin{array}{c}\text { Major } \\
{\left[{ }^{\prime \prime}\right]}\end{array}$ & Ratio & $\begin{array}{c}\text { Major } \\
{\left[{ }^{\prime \prime}\right]}\end{array}$ & Ratio & \\
\hline \multicolumn{7}{|c|}{ M-type semi-regular and irregular stars: } \\
\hline $\mathrm{L}_{2}$ Pup & 10.6 & $6.04 \pm 0.08$ & $0.85 \pm 0.02$ & 5.9: & 1.0: & $2,5,8$ \\
\hline W Hya & 22.6 & $10.92 \pm 0.12$ & $0.75 \pm 0.06$ & $5.59 \pm 0.06$ & $0.89 \pm 0.01$ & \\
\hline T Mic & 12.0 & $7.04 \pm 0.12$ & $0.99 \pm 0.02$ & $3.48 \pm 0.08$ & $0.97 \pm 0.05$ & 6,7 \\
\hline Y Scl & 6.0 & $3.88 \pm 0.20$ & $0.89 \pm 0.05$ & $3.02 \pm 0.16$ & $0.75 \pm 0.05$ & 8 \\
\hline V1943 Sgr & 13.0 & $6.95 \pm 0.12$ & $0.94 \pm 0.03$ & 7.9: & 0.9: & 2 \\
\hline BK Vir & 12.2 & $5.84 \pm 0.15$ & $0.89 \pm 0.04$ & $3.46 \pm 0.04$ & $0.90 \pm 0.02$ & 5,6 \\
\hline V Tel & 8.0 & $5.00 \pm 0.08$ & $0.95 \pm 0.02$ & $3.22 \pm 0.07$ & $0.94 \pm 0.03$ & 1 \\
\hline SU Vel & 9.8 & $4.85 \pm 0.07$ & $0.76 \pm 0.01$ & $3.37 \pm 0.05$ & $0.75 \pm 0.02$ & \\
\hline UY Cet & 9.0 & $5.48 \pm 0.13$ & $0.82 \pm 0.03$ & $3.04 \pm 0.08$ & $0.86 \pm 0.03$ & 4 \\
\hline SV Aqr & 5.8 & $3.83 \pm 0.17$ & $0.94 \pm 0.06$ & $2.36 \pm 0.18$ & $0.98 \pm 0.14$ & 6 \\
\hline SW Vir & 27.0 & $8.92 \pm 0.13$ & $1.00 \pm 0.02$ & 6.7: & 1.0: & 2,6 \\
\hline CW Cnc & 12.6 & $8.00 \pm 0.15$ & $0.88 \pm 0.02$ & $3.92 \pm 0.13$ & $0.92 \pm 0.04$ & 7 \\
\hline RT Vir & 21.2 & $8.18 \pm 0.14$ & $0.89 \pm 0.02$ & $5.25 \pm 0.06$ & $0.82 \pm 0.01$ & 7 \\
\hline $\mathrm{R} \mathrm{Crt}$ & 25.0 & $7.72 \pm 0.08$ & 1.0: & 5.5: & $0.9:$ & 2 \\
\hline \multicolumn{7}{|c|}{ M-type Mira stars: } \\
\hline R Leo & 18.2 & $10.00 \pm 0.11$ & $0.88 \pm 0.02$ & $4.70 \pm 0.08$ & 1.0: & $2,5,8$ \\
\hline R Hya & 18.8 & 4.5: & 1.0: & 4.5: & 0.8: & $6,7,8$ \\
\hline R Hor & 16.8 & $7.28 \pm 0.04$ & $0.84 \pm 0.01$ & 4.1: & 0.8: & 2,4 \\
\hline RR Aql & 4.5 & $6.39 \pm 0.07$ & $0.95 \pm 0$ & 7.2: & $0.9:$ & 2,5 \\
\hline IRC-10529 & 28.6 & $7.00 \pm 0.06$ & $0.93 \pm 0.01$ & 8.2: & 0.6 : & 2,3 \\
\hline WX Psc & 25.4 & 11.6: & 0.9: & 8.5: & $0.7:$ & 2,3 \\
\hline IRC +10365 & 40.8 & $7.73 \pm 0.08$ & $0.93 \pm 0.01$ & $4.18 \pm 0.05$ & $0.92 \pm 0.02$ & 3 \\
\hline \multicolumn{7}{|c|}{ C-type semi-regular and irregular stars: } \\
\hline TW Oph & 8.0 & $4.68 \pm 0.26$ & $1.00 \pm 0.08$ & $2.57 \pm 0.09$ & $0.96 \pm 0.04$ & \\
\hline NP Pup & 5.8 & $4.63 \pm 0.27$ & $0.93 \pm 0.08$ & $3.16 \pm 0.32$ & $0.87 \pm 0.12$ & 3 \\
\hline TW Hor & 8.4 & $5.77 \pm 0.06$ & $0.66 \pm 0.01$ & $2.65 \pm 0.05$ & $0.94 \pm 0.02$ & 5,6 \\
\hline T Ind & 5.6 & $3.50 \pm 0.14$ & $0.83 \pm 0.06$ & $2.14 \pm 0.28$ & $0.91 \pm 0.13$ & 1,3 \\
\hline RT Cap & 7.0 & $4.42 \pm 0.23$ & $0.89 \pm 0.05$ & $2.25 \pm 0.20$ & $1.00 \pm 0.15$ & 1 \\
\hline AQ Sgr & 9.0 & $4.93 \pm 0.11$ & $0.90 \pm 0.02$ & $1.4:$ & 1.0: & \\
\hline U Hya & 17.8 & 10.8: & 0.8: & 6.2: & $0.7:$ & 2 \\
\hline W Ori & 9.2 & $4.38 \pm 0.07$ & $0.98 \pm 0.03$ & $3.28 \pm 0.07$ & $0.92 \pm 0.02$ & 7,8 \\
\hline V Aql & 10.6 & $5.65 \pm 0.09$ & $0.97 \pm 0.02$ & $3.22 \pm 0.05$ & $0.94 \pm 0.02$ & \\
\hline Y Pav & 11.2 & $4.89 \pm 0.10$ & $0.99 \pm 0.03$ & $3.04 \pm 0.06$ & $0.94 \pm 0.03$ & \\
\hline X Vel & 13.0 & $4.07 \pm 0.10$ & $0.99 \pm 0.03$ & $2.63 \pm 0.09$ & $0.92 \pm 0.04$ & 3 \\
\hline Y Hya & 12.2 & $5.39 \pm 0.15$ & 1.0: & $2.63 \pm 0.10$ & $0.89 \pm 0.06$ & \\
\hline SS Vir & 7.4 & $5.88 \pm 0.86$ & $0.57 \pm 0.13$ & $3.34 \pm 0.25$ & $0.70 \pm 0.08$ & $5,6,8$ \\
\hline W CMa & 11.6 & $4.26 \pm 0.11$ & $1.00 \pm 0.02$ & $3.20 \pm 0.08$ & $0.98 \pm 0.03$ & 6 \\
\hline \multicolumn{7}{|c|}{ C-type Mira stars: } \\
\hline R Lep & 17.2 & 11.7: & 0.9: & $3.58 \pm 0.07$ & $0.99 \pm 0.03$ & 2 \\
\hline CZ Hya & 9.8 & $3.98 \pm 0.11$ & $0.99 \pm 0.04$ & $2.47 \pm 0.15$ & $0.88 \pm 0.09$ & \\
\hline R For & 15.8 & $5.69 \pm 0.11$ & $0.99 \pm 0.02$ & $3.60 \pm 0.07$ & $0.93 \pm 0.03$ & 3 \\
\hline R Vol & 16.6 & $5.83 \pm 0.08$ & $0.97 \pm 0.02$ & 4.0: & 1.0: & 2,3 \\
\hline RV Aqr & 24.6 & $6.01 \pm 0.05$ & $0.99 \pm 0.01$ & 4.2: & $0.9:$ & 2,3 \\
\hline V688 Mon & 16.3 & 6: & 1: & $2.98 \pm 0.08$ & $0.97 \pm 0.03$ & 3 \\
\hline V1259 Ori & 20.9 & 5.0: & 1.0: & $3.48 \pm 0.06$ & $0.92 \pm 0.02$ & 3,4 \\
\hline
\end{tabular}

Notes. The second column gives the photodissociation radius two times over the distance as a measure of the expected size of the $\mathrm{CO}$ emitting region. Columns 3-6 give the major axis and the axis ratio with errors for the best-fit Gaussian at the center velocity of the respective line. The final column gives the asymmetrical features for each source, as is explained in Sect. 4.3. See text for a further explanation.

the LIME 1.9.5 radiative transfer code (Brinch \& Hogerheijde $2010)$ to produce radiative transfer models of the photodissociation models from Saberi et al. (2019), using the CSE parameters that were adopted in their chemical models. Subsequently, we fit a Gaussian profile to the $\mathrm{CO}(2-1)$ emission at the systemic velocity using a $1 \mathrm{~km} \mathrm{~s}^{-1}$ channel width using the same method as in Sect. 3.2. Figure 3 shows the $\mathrm{CO}(2-1)$ line intensity distributions for three M-star models with different mass-loss rates. Each model intensity distribution has been scaled to the fitted Gaussian. For intermediate- to high-mass-loss-rate sources

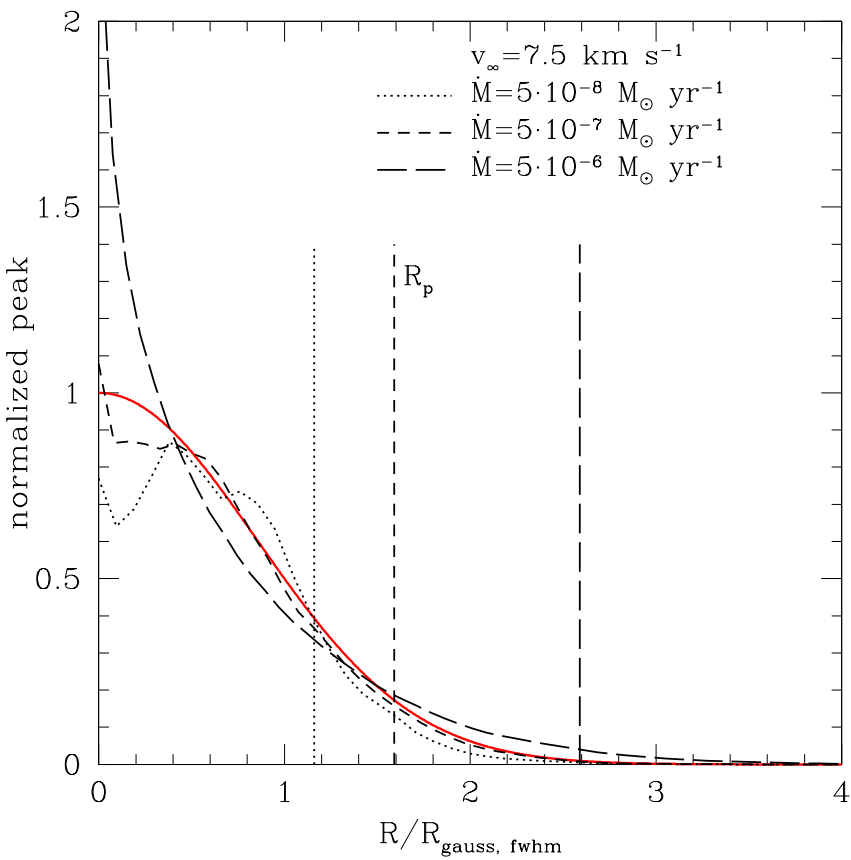

Fig. 3. $\mathrm{CO}(2-1)$ intensity distribution at the systemic velocity as a function of radius for three M-star models from Saberi et al. (2019). The models were normalized to the peak of the fitted Gaussian and scaled to the FWHM radius of that Gaussian for each model separately. The normalized Gaussian is indicated by the solid red line in the figure. For each model, the vertical lines indicate the photodissociation radius $R_{\mathrm{p}}$, which was also scaled to the Gaussian FWHM radius for each model. See text for a further explanation.

in particular, the intensity distribution is significantly nonGaussian, and the determination of a Gaussian FWHM is strongly dependent on the exact shape of the intensity distribution in the inner region. Since low-angular-resolution observations lack the uv-coverage to extract this information and as irregular structures are especially common in these areas, the Gaussian radii determined for the CSEs with the highest density, $\dot{M} / v_{\infty} \gtrsim 10^{-7} M_{\odot} \mathrm{km} \mathrm{s}^{-1} \mathrm{yr}^{-1}$, are less reliable. The figure also shows the calculated photodissociation radius from Saberi et al. (2019) for each model (vertical lines) scaled with the Gaussian FWHM radius. For the densest CSEs, the $\mathrm{CO}(2-1)$ line does not extend to the photodissociation radius and the Gaussian radii are therefore not a measure for $R_{\mathrm{p}}$.

Based on the formula and the distance estimates from Stanek et al. (1995) in Table 1, the $\mathrm{CO}(2-1)$ major axis is a factor of two smaller than $2 \times R_{\mathrm{p}} / D$ for the semi- and irregular variables, and a factor of three smaller for the Mira variables, regardless of chemical type. This is consistent with the expectations from the modeling (both chemical and radiative transfer). Furthermore, for a small majority of the sources (26/42), the $\mathrm{CO}(2-1)$ axis ratio deviates $\leq 10 \%$ from one, meaning that slightly more than half of the sources are close to being circular. For the more aspherical sources, the photodissociation calculations that assume a standard spherically expanding CSE introduce further uncertainties.

The major axes of the Gaussian fits to the $\mathrm{CO}(2-1)$ emission at the center velocity channels (third column of Table 2 multiplied with the distance from Table 1) are plotted against $\dot{M} / \nu_{\infty}$ in Fig. 4. The uncertainty of the major axis is very small in arcseconds (see Figs. E.1-E.2). Instead, the error bars in the $y$-direction are dominated by the distance uncertainty, which cannot be evaluated easily. The figure also shows the photodissociation 


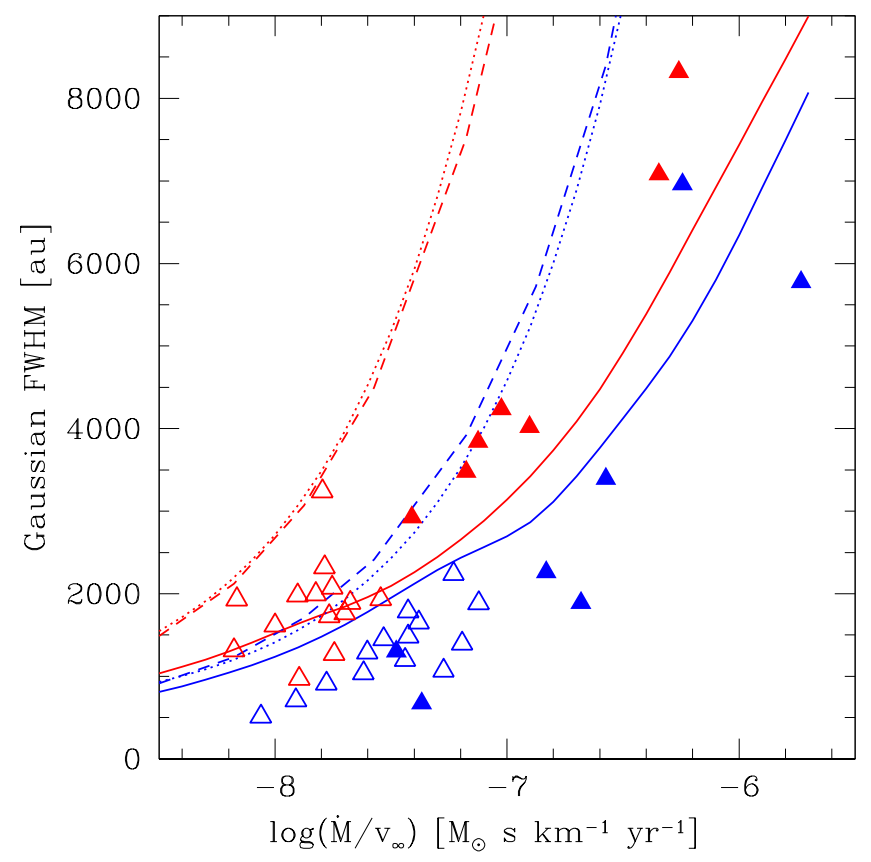

Fig. 4. Full-width half-maximum (FWHM) major axis of the bestfit Gaussian at the center velocity channel of the $\mathrm{CO}(2-1)$ emission (marked by triangles and in astronomical units by multiplying by the distance from Table 1) as a function of circumstellar density (as measured by $\dot{M} / v_{\infty}$ from Table 1$)$. M-type stars are blue. C-type stars are red. Mira-type variables are marked with solid symbols and other variables appear with open symbols. The dashed lines show the photodissociation diameter for M-type (blue) and C-type (red) stars from the model grid by Saberi et al. (2019) (calculated with $v_{\infty}=7.5 \mathrm{~km} \mathrm{~s}^{-1}$ ). The dotted lines are the parametrized fits to the results from Mamon et al. (1988). The solid lines show a spline-fit to the expected Gaussian FWHM determined from radiative transfer modeling of the models from Saberi et al. (2019). See text for a further explanation.

diameter for the M-type and C-type stars, respectively. The photodissociation radii were calculated across the range of $\dot{M} / v_{\infty}$ for $v_{\infty}=7.5 \mathrm{~km} \mathrm{~s}^{-1}$. We used the LIME 1.9 .5 radiative transfer code again (Brinch \& Hogerheijde 2010) to produce radiative transfer models of the Saberi et al. (2019) photodissociation model grid, and we used the same Gaussian fitting procedure as mentioned above to measure the predicted $\mathrm{CO}(2-1)$ size. A spline fit to the expected $\mathrm{CO}(2-1)$ size is shown by the solid lines in Fig. 4, which thus indicates the dependence of the measured size of the $\mathrm{CO}(2-1)$ emitting region on the photodissociation radius as well as on the circumstellar density. The different results for higher- and lower-mass-loss-rate sources are apparent in the figure: the measured size of the $\mathrm{CO}(2-1)$ emitting region for lower-mass-loss-rate M-type stars appears to show a rather weak dependence on the circumstellar density, which is also seen in the dependence of photodissociation diameter and the modeled Gaussian diameter on the density. For higher-mass-loss rate M-type Mira sources, the dependence on the density is much steeper, with a slope similar to what is expected from the photodissociation models. In almost all cases, however, the measured diameter is smaller than the expected $\mathrm{CO}(2-1)$ diameter based on the chemical and radiative transfer models. The lower-mass-loss-rate C-type stars show no significant dependence on circumstellar density, or at least a large scatter around the expected relation. Essentially all of the observed low-mass-loss-rate C-type stars have $\mathrm{CO}(2-1)$-emitting major axes of 1000-2000 AU. The higher-mass-loss-rate C-type Mira stars, on the other hand, show a dependence on density that appears to be somewhat steeper than for the M-type Miras. For all C-type Miras, the measured diameters are larger than expected.

Although the measured size of the $\mathrm{CO}(2-1)$ region is smaller than the calculated photodissociation diameter for essentially all sources, it is apparent from Fig. 4 that the correlation between the two is not straightforward. There are a variety of possible explanations for the deviations between the observations and the photodissociation models. The most obvious one is the uncertainty of the distance, which introduces significant scatter in the size determinations. Furthermore, as shown in Saberi et al. (2019), changes in initial CO abundance, CSE temperature profiles, and the interstellar radiation field can have significant effects. Finally, different density profiles, dust properties, or dust-to-gas ratios from what is assumed in the photodissociation models can all change the estimated radius.

The full results of the fitting of Gaussian emission distributions to the ALMA ACA data across all of the CO line channels are displayed in Figs. E.1-E.2. The major and minor axis of each channel, as well as the RA and Dec offsets of the center of the best-fit Gaussian relative to the SIMBAD J2000 coordinates of each source, are plotted against lsr-velocity for each line and source as denoted in each plot. The interpretation of these plots is further discussed in Sect. 4.3.

\subsubsection{Comparison with results from spherically symmetric radiative transfer models}

Here we present the comparison between the emission distributions from ALMA and those calculated from our previous bestfit radiative transfer model results (Sect. 3.3). The same analysis was performed on the model results as on the observational data. Figure 5 shows the results of the Gaussian emission distribution fitting (Sects. 3.2 and 4.2.1) from the models compared with the observational results for the $\mathrm{CO}(2-1)$ emission for each of the six selected sources. For error bars on the observational results, see Figs. E.1-E.2. The results are affected by the difficulties of fitting a Gaussian distribution to the weaker and smaller emission distribution close to the edge of the line. It is obvious that the radiative transfer analysis of the full data set will be necessary before any firm conclusions can be drawn. For the M-type stars, it seems as if the size of the emission might be smaller than expected for the low mass-loss-rate source (R Leo) and the opposite for the high mass-loss-rate source (IRC-10529). The upcoming analysis will evaluate whether this is a general trend or specific to the selected sources. This trend would indicate an even steeper dependence on the circumstellar density than predicted from the photodissociation model by Mamon et al. (1988) and is contrary to the fit to the M-type Mira stars in Fig. 4. The results for the C-type stars are less clear, partly because the fitting results to the $\mathrm{U}$ Hya data seem strongly affected by resolved out flux (see Sect. 4.3). It is also possible that the emission from C-type stars is subject to stronger optical depth effects due to the higher $\mathrm{CO}$ abundance. This will also be evaluated in the upcoming radiative transfer analysis.

\subsection{Asymmetrical features}

Several asymmetrical features can be identified in the observational results presented thus far, that is, the line profiles (Figs. D.1-D.2) and the results from the Gaussian fitting (Figs. E.1-E.2). These features can be divided into two classes: 

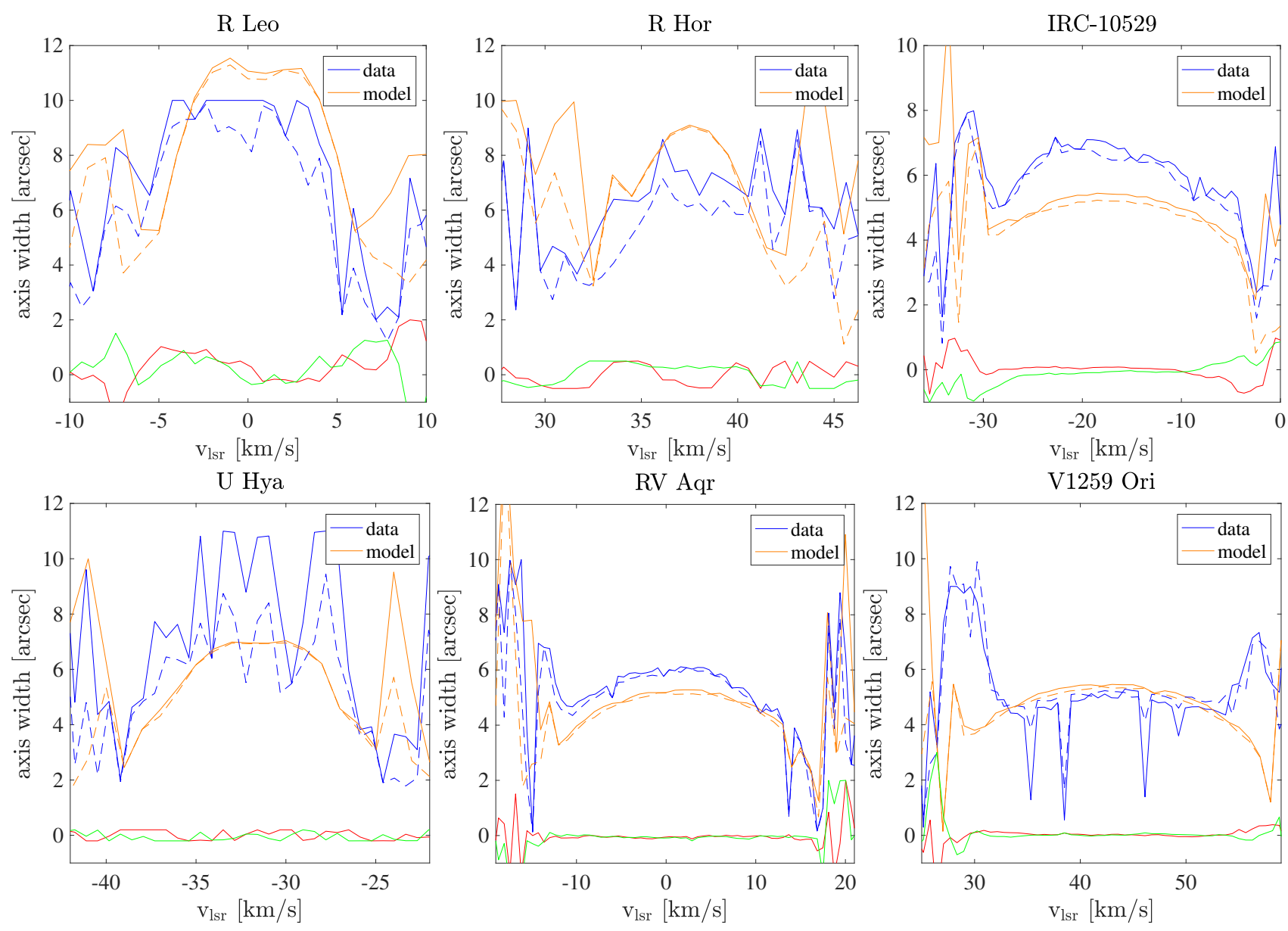

Fig. 5. Comparison between the results from fitting a Gaussian emission distribution to the $\mathrm{CO}(2-1)$ data (blue) and the model results (orange) based on previous best-fit models (see text). The solid lines show the major axis and the dashed lines show the minor axis. The red and green lines show the RA and Dec. offsets for the data, respectively. Top row: three M-type stars, and bottom row: three C-type stars, with an increasing mass-loss rate (Table 1). The results are affected by the difficulties of fitting a Gaussian distribution to the weaker and smaller emission distribution close to the edge of the line. For error bars on the results from the measurements, see Figs. E.1-E.2.

Features that are consistent with a spherically symmetric CSE and those that are not.

\subsubsection{Consistent with a smooth outflow}

The features that can be explained with a spherically symmetric CSE are as follows.

1. An offset between the center position (of the fitted Gaussian) and the source coordinates is explained the most easily by uncertainties in the adopted coordinates (taken from SIMBAD pre-Gaia DR2), when seen consistently in both lines. Examples include V Tel and RT Cap, for instance.

2. The interferometer acts as a spatial filter and since only the ACA was used (and not the total-power array), largescale, smooth emission is resolved out. Resolved-out flux results in a lower peak flux level than when measured with a (similar-sized) single-dish telescope. Furthermore, if a significant fraction of the flux is resolved out, the visibility distribution cannot be well-fitted by a simple Gaussian distribution. This is the most apparent across the center channels where the emission distribution is expected to be the largest. Therefore, it is also a more common problem for the $\mathrm{CO}(3-2)$ line where the beam is smaller. Examples of this can be seen in RR Aql (CO(3-2)), WX Psc (both lines), and $\mathrm{R}$ Vol $(\mathrm{CO}(3-2))$.
3. Self-absorption on the blue-shifted side of the line can be seen as asymmetry in the line profile where it is sometimes apparent that emission is "missing" on the blue side of the line. Examples include IRC-10529, NP Pup, and T Ind. It is even more apparent when looking at how the sizes of the sources change across the channels. Sources with selfabsorption show a prominent peak in the size distribution at blue-shifted velocities since these channels probe emission coming from cooler circumstellar layers (and hence a larger region).

These features are relatively easy to explain and related to how the observations were performed for the first and second points.

\subsubsection{Indications of circumstellar anisotropic structure}

The next class of features is less straight-forward to interpret and the features are mostly thought to be clearly inconsistent with a spherically symmetric outflow. Further modeling and/or observations will be necessary to explain exactly how they arise. The majority of sources that exhibit these features need to be followed up with higher-spatial-resolution observations. Features that cannot be explained by a smoothly expanding, spherically symmetric CSE are the following.

4. Triangular line profiles are not a newly observed feature; however, a satisfactory explanation as to how they arise 
is lacking. This feature seems to be more common among high-mass-loss-rate sources. Clear examples are R Hor and V1259 Ori.

5. High-velocity wings are not a new feature either, but they can be detected from more sources in this data set due to the superior sensitivity reached with the ACA observations compared to previous single-dish observations. The line profiles of TW Hor, for example, are very similar to those of the known asymmetrical CSE of $\pi^{1}$ Gru (e.g., Doan et al. 2020). The CO emission from $\pi^{1}$ Gru can be reproduced by a CSE consisting of an expanding equatorial torus (explaining the two central peaks) and a bi-polar faster outflow leading to wide wings (Doan et al. 2017). Other, more tentative examples of line profiles with high-velocity wings include those of $\mathrm{L}_{2}$ Pup, R Leo, and BK Vir.

6. The fitting of the visibility distribution gives the center position of the best-fit Gaussian in each channel and the offset relative to the center position is plotted in Figs. E.1-E.2. Several sources show center position gradients. A position gradient with velocity is either indicative of expansion in a higher-density layer (e.g., an equatorial torus such as in the case of $\pi^{1} \mathrm{Gru}$ ) or of rotation (e.g., Ramstedt et al. 2018; Vlemmings et al. 2018) depending on the orientation of the sources. Examples of sources with center position gradients are BK Vir (in RA), TW Hor (both RA and Dec), SS Vir (both RA and Dec), and W CMa (in RA).

7. A spherically symmetric CSE with a constant expansion velocity grows monotonically in size from the edge of the line to the center velocity. The effects of self-absorption and resolved-out flux on the size distribution are discussed above. For some sources, the source size changes in an unpredictable way that cannot be easily explained without further modeling and/or observations. Examples include T Mic, CW Cnc, RT Vir, and W Ori.

8. It is directly apparent from the line profile that the emission cannot come from a standard CSE for a handful of the sample sources. Instead, these line profiles are anomalous. Some are previously known to be anomalous, for example, $\mathrm{L}_{2}$ Pup (e.g., Kervella et al. 2016) and R Leo (Fonfría et al. 2019), while others have not been studied with a focus on the detailed structure of the CSE, for example, R Hya and SS Vir.

\subsection{Detections of emission from molecules other than ${ }^{12} \mathrm{CO}$}

A large number of molecules other than ${ }^{12} \mathrm{CO}$ have transitions with frequencies within the observed bands. For all sources, the peak fluxes of the detected lines as measured across a 10" aperture are listed in Tables C.1-C.4. The spectral resolution across each spectral window is the same as the one used for imaging and is given in Sect. 3. The lines listed in Tables C.1-C.4 are those for which we are confident of detection at the given spectral resolution and aperture. It is possible that further detections could be confirmed with more optimization of the data analysis.

Lines from $\mathrm{SiO}, \mathrm{SiS},{ }^{13} \mathrm{CO}, \mathrm{CS}$, and $\mathrm{H}^{13} \mathrm{CN}$ are detected in a majority of the sources. Unsurprisingly, oxides such as $\mathrm{SO}$, $\mathrm{SO}_{2}$, and $\mathrm{H}_{2} \mathrm{O}$ are only detected in the M-type stars as are $\mathrm{H}_{2} \mathrm{~S}$ and less common isotopologues of $\mathrm{SiS}$ and $\mathrm{NaCl}$. In the C-type stars, ${ }^{13} \mathrm{CN}$ and ${ }^{13} \mathrm{CS}$ are detected, sometimes together with lines from molecules with several carbon atoms, such as $\mathrm{HC}_{3} \mathrm{~N}, \mathrm{C}_{4} \mathrm{H}$, and $\mathrm{SiC}_{2}$. A further analysis of these lines is beyond the scope of this paper, but an overview of the firm detections are given here and follow-up studies are encouraged.

\section{Discussion and summary}

The measurements presented in this paper show that, for this sample, the $\mathrm{CO}$ envelope sizes are, in general, larger for C-type than for M-type stars. As explained both above and below, the envelope size depends on many different parameters, but this trend is expected if the $\mathrm{CO} / \mathrm{H}_{2}$ ratio is larger in C-type stars. Based on chemical equilibrium models, the ratio between the $\mathrm{C}$ and M-type CO-abundance is generally assumed to be equal to five when deriving total gas mass-loss rates from observations of $\mathrm{CO}$ emission lines. As part of the analysis planned for the full DEATHSTAR sample, the difference in abundance depending on chemical type (also S-type stars) can now be observationally constrained for a large sample of AGB stars.

Furthermore, the measurements show a relation between the measured (Gaussian) $\mathrm{CO}(2-1)$ size and circumstellar density that, while in broad agreement with photodissociation calculations, reveal large scatter and some systematic differences between the different stellar types. A significant amount of scatter arises due to significant distance uncertainties. Part of the differences can also be explained by excitation and optical depth effects that decrease the reliability of the Gaussian size determination for $\dot{M} / v_{\infty} \gtrsim 10^{-7} M_{\odot} \mathrm{km} \mathrm{s}^{-1} \mathrm{yr}^{-1}$. For lower-massloss-rate irregular and semi-regular variables of both $\mathrm{M}$ - and C-type stars, the $\mathrm{CO}(2-1)$ size seems essentially independent of $\dot{M} / v_{\infty}$. For the higher-mass-loss-rate Mira stars, the $\operatorname{CO}(2-1)$ size clearly increases with circumstellar density, with larger sizes for the higher CO-abundance C-type stars. The M-type stars appear to be consistently smaller than what was predicted from photodissociation theory. The differences between the estimates and measurements could (as shown in Saberi et al. 2019) be due to, for example, a systematically overestimated $\mathrm{CO}$ abundance, differences in the CSE temperature profile, or differences in the UV-environment. Additionally, a difference in the adopted properties of the dust, which is responsible for much of the shielding in low density CSEs, and/or the adopted dust-to-gas ratio in the models would lead to different sizes. Finally, a difference in the CSE density profile due to changes in velocity and/or the mass loss rate could significantly alter the photodissociation radius. With the size measurements that are now available for the large sample of sources in the DEATHSTAR project, it will be possible to investigate many of these factors in more detail.

In recent years there has been a strong focus on investigating the isotropy of the mass-loss process in low- to intermediate mass evolved stars, which was partly expedited by the superior imaging capabilities of ALMA. Several AGB sources with previously known complex circumstellar dynamics and/or binary companions have been mapped in detail (e.g., Ramstedt et al. 2014; Decin et al. 2015; Maercker et al. 2016; Doan et al. 2017; Kim et al. 2017; Homan et al. 2018; Fonfría et al. 2019). With the DEATHSTAR project, the aim is to get an overview of how widespread and significant the detected asymmetrical features are, from the perspective of measuring the amount of circumstellar wind material. A goal is to eventually be able to evaluate the uncertainties that these features will result in when estimating mass-loss rates from more distant, unresolved sources.

The results presented here show that the majority of the sources have CO CSEs that are consistent with a spherically symmetric, smooth outflow, at least on larger scales. This is based on the line profile shapes and that the emission distribution across the line channels can be well-fitted with a Gaussian visibility distribution with axis ratios that are close to one for a majority of the sources. For about a third of the sources, indications of strong asymmetries are detected. This is consistent 
with what was found in previous interferometric investigations of northern sources (Neri et al. 1998; Castro-Carrizo et al. 2010). In the DEATHSTAR sample, some of the known asymmetric sources have been removed, but the higher sensitivity reached in the ACA observations allowed us to detect relatively weaker features, such as extended line wings, than what was possible in previous investigations. This offers an explanation as to why the same fraction of strong asymmetries is still found. In a large fraction of the sources, some deviation from spherical symmetry is detected, often on smaller scales. Whether these smaller deviations can have a significant effect on the estimated mass-loss rate or not cannot be evaluated without further analysis.

The peak fluxes of lines from molecules other than ${ }^{12} \mathrm{CO}$ detected within the observed bands are listed in Tables C.1-C.4. In general, lines from molecules that are known to be abundant in AGB CSEs (e.g., $\mathrm{SiO}, \mathrm{SiS}, \mathrm{CS}$ ) are detected in almost all sources. Some less abundant oxides (e.g., $\mathrm{H}_{2} \mathrm{O}$ and $\mathrm{SO}$ ) are only detected in the M-type stars, while emission from molecules with several carbon atoms (e.g., $\mathrm{SiC}_{2}$ ) are only detected toward the C-type stars. No further analysis has been attempted.

\section{Outlook}

This paper presents the first observational results from the DEATHSTAR project. The $\mathrm{CO}(2-1)$ and (3-2) ALMA ACA observations of the southern $\mathrm{M}$ - and C-type stars observed in ALMA Cycle 4 are presented and analyzed. In an upcoming publication, the results on the M-, S-, and C-type sources observed in Cycle 5 will be presented. The next step is to perform detailed radiative transfer modeling that is constrained by the presented ACA data together with the previously attained single-dish observations of the lower- $J$ CO transition lines (up to $J=6 \rightarrow 5$ ) in order to determine mass-loss rates that are independent of photodissociation model results. This will also be presented in future publications. For a large fraction of the sources, observations at higher spatial resolution will be necessary to deduce the nature and origin of the complex circumstellar dynamics revealed by the observations and data analysis presented in this paper.

Acknowledgements. This paper makes use of the following ALMA data ADS/JAO.ALMA\#2016.2.00025.S and \#2017.1.00595.S. ALMA is a partnership of ESO (representing its member states), NSF (USA) and NINS (Japan), together with NRC (Canada), MOST and ASIAA (Taiwan), and KASI (Republic of Korea), in cooperation with the Republic of Chile. The Joint ALMA Observatory is operated by ESO, AUI/NRAO and NAOJ. This work has made use of data from the European Space Agency (ESA) mission Gaia (https://www cosmos.esa.int/gaia), processed by the Gaia Data Processing and Analysis Consortium (DPAC, https://www. cosmos.esa.int/web/gaia/dpac/ consortium). Funding for the DPAC has been provided by national institutions, in particular the institutions participating in the Gaia Multilateral Agreement.

\section{References}

Bailer-Jones, C. A. L., Rybizki, J., Fouesneau, M., Mantelet, G., \& Andrae, R. 2018, AJ, 156, 58

Bladh, S., Höfner, S., Aringer, B., \& Eriksson, K. 2015, A\&A, 575, A105

Bladh, S., Liljegren, S., Höfner, S., Aringer, B., \& Marigo, P. 2019, A\&A, 626 A100
Brinch, C., \& Hogerheijde, M. R. 2010, A\&A, 523, A25

Castro-Carrizo, A., Quintana-Lacaci, G., Neri, R., et al. 2010, A\&A, 523, A59

Chiavassa, A., Freytag, B., \& Schultheis, M. 2018, A\&A, 617, L1

Conroy, C. 2013, ARA\&A, 51, 393

Danilovich, T., Teyssier, D., Justtanont, K., et al. 2015, A\&A, 581, A60

Decin, L., Richards, A. M. S., Neufeld, D., et al. 2015, A\&A, 574, A5

Doan, L., Ramstedt, S., Vlemmings, W. H. T., et al. 2017, A\&A, 605, A28

Doan, L., Ramstedt, S., Vlemmings, W. H. T., et al. 2020, A\&A, 633, A13

Draine, B. T. 1978, ApJS, 36, 595

Eriksson, K., Nowotny, W., Höfner, S., Aringer, B., \& Wachter, A. 2014, A\&A, 566, A95

ESA 1997, The HIPPARCOS and TYCHO Catalogues. Astrometric and Photometric Star Catalogues Derived from the ESA HIPPARCOS Space Astrometry Mission, 1200

Fonfría, J. P., Santander-García, M., Cernicharo, J., et al. 2019, A\&A, 622, L14

Gaia Collaboration (Prusti, T., et al.) 2016, A\&A, 595, A1

Gaia Collaboration (Brown, A. G. A., et al.) 2018, A\&A, 616, A1

González Delgado, D., Olofsson, H., Kerschbaum, F., et al. 2003, A\&A, 411, 123

Groenewegen, M. A. T. 2017, A\&A, 606, A67

Groenewegen, M. A. T., \& Whitelock, P. A. 1996, MNRAS, 281, 1347

Höfner, S. 2008, A\&A, 491, L1

Höfner, S., \& Olofsson, H. 2018, A\&ARv, 26, 1

Höfner, S., Bladh, S., Aringer, B., \& Ahuja, R. 2016, A\&A, 594, A108

Homan, W., Richards, A., Decin, L., de Koter, A., \& Kervella, P. 2018, A\&A, 616, A34

Ivezic, Z., Nenkova, M., \& Elitzur, M. 1999, ArXiv e-prints [arXiv:astro-ph/9910475]

Jura, M. 1990, in From Miras to Planetary Nebulae: Which Path for Stellar Evolution?, eds. M. O. Mennessier, \& A. Omont, 41

Jura, M., \& Kleinmann, S. G. 1990, ApJ, 364, 663

Jura, M., \& Kleinmann, S. G. 1992, ApJS, 79, 105

Jura, M., Yamamoto, A., \& Kleinmann, S. G. 1993, ApJ, 413, 298

Kervella, P., Homan, W., Richards, A. M. S., et al. 2016, A\&A, 596, A92

Kim, H., Trejo, A., Liu, S.-Y., et al. 2017, Nat. Astron., 1, 0060

Lindegren, L., Lammers, U., Hobbs, D., et al. 2012, A\&A, 538, A78

Maercker, M., Vlemmings, W. H. T., Brunner, M., et al. 2016, A\&A, 586, A5

Mamon, G. A., Glassgold, A. E., \& Huggins, P. J. 1988, ApJ, 328, 797

Marigo, P., Ripamonti, E., Nanni, A., Bressan, A., \& Girardi, L. 2016, MNRAS, 456, 23

Martí-Vidal, I., Vlemmings, W. H. T., Muller, S., \& Casey, S. 2014, A\&A, 563, A136

Matsuno, M., Nakagawa, A., Morita, A., et al. 2020, PASJ, 72, 56

McMullin, J. P., Waters, B., Schiebel, D., Young, W., \& Golap, K. 2007, in Astronomical Data Analysis Software and Systems XVI, eds. R. A. Shaw, F. Hill, \& D. J. Bell, ASP Conf. Ser., 376, 127

Menzies, J. W., Feast, M. W., \& Whitelock, P. A. 2006, MNRAS, 369, 783

Neri, R., Kahane, C., Lucas, R., Bujarrabal, V., \& Loup, C. 1998, A\&AS, 130, 1

Ramstedt, S., \& Olofsson, H. 2014, A\&A, 566, A145

Ramstedt, S., Schöier, F. L., Olofsson, H., \& Lundgren, A. A. 2006, A\&A, 454, L103

Ramstedt, S., Schöier, F. L., Olofsson, H., \& Lundgren, A. A. 2008, A\&A, 487, 645

Ramstedt, S., Schöier, F. L., \& Olofsson, H. 2009, A\&A, 499, 515

Ramstedt, S., Mohamed, S., Vlemmings, W. H. T., et al. 2014, A\&A, 570, L14

Ramstedt, S., Mohamed, S., Olander, T., et al. 2018, A\&A, 616, A61

Saberi, M., Vlemmings, W. H. T., \& De Beck, E. 2019, A\&A, 625, A81

Samus', N. N., Kazarovets, E. V., Durlevich, O. V., Kireeva, N. N., \& Pastukhova, E. N. 2017, Astron. Rep., 61, 80

Schöier, F. L. 2000, PhD Thesis, Stockholm Observatory, SE-133 36, Saltsjöbaden, Sweden

Schöier, F. L., \& Olofsson, H. 2001, A\&A, 368, 969

Stanek, K. Z., Knapp, G. R., Young, K., \& Phillips, T. G. 1995, ApJS, 100, 169

van Langevelde, H., Quiroga-Nuñez, L. H., Vlemmings, W. H. T., et al. 2018, 14th European VLBI Network Symposium \& Users Meeting (EVN 2018), 43

Vlemmings, W. H. T., Khouri, T., De Beck, E., et al. 2018, A\&A, 613, L4

Whitelock, P., Menzies, J., Feast, M., et al. 1994, MNRAS, 267, 711 


\section{Appendix A: Parallaxes for AGB stars from Gaia DR2}

Since the paper presents mass-loss values based on earlier analysis, we have presented the distances that were used in the massloss determinations. Recently, Gaia DR2 astrometric solutions were released for all but one of the stars in our sample. While the formal errors on the Gaia parallaxes are generally small, the reliability of the Gaia parallaxes for AGB stars is under debate (e.g., van Langevelde et al. 2018). There are several reasons why the Gaia parallaxes to AGB stars might be wrong or have significantly underestimated the assigned errors. Firstly, AGB stars are often larger than the parallax signature itself (with sizes of order one to a few astronomical units) and they have convective surface motions that can cause the photo-center to shift significantly (Chiavassa et al. 2018). Secondly, AGB pulsations can affect the astrometric measurements. Finally, AGB stars are so bright that Gaia approaches saturation. The Gaia DR2 catalog contains a number of parameters that can provide a measure for the reliability of the astrometry. One of these is the astrometric_excess_noise, which represents modeling errors for sources that do not behave according to the adopted astrometric model of Lindegren et al. (2012). In van Langevelde et al. (2018), an empirical analysis of very long baseline interferometry (VLBI) parallaxes compared to the Gaia results revealed that adding the astrometric_excess_noise to the formal parallax errors in quadrature was needed to reconcile the two methods. In Fig. A.1 we show the difference between the formal and excess noise errors. When we adopted this procedure, only four of the AGB stars in our sample have a parallax solution that satisfies $\pi / \sigma_{\pi}>5$. Recently, another measure of reliability was introduced, namely the RUWE $^{3}$ (renormalized unit weighted error). This represents the square-root of the reduced $\chi^{2}$ value, which was corrected for the strong dependence of the $\chi^{2}$ value on magnitude and color. For many applications, a RUWE value $<1.4$ is empirically found to represent a good fit. However, as is noted in Lindegren et al. (2018), the RUWE normalization does not work optimally for the brightest sources $G<12$, which includes all of our AGB stars. In these cases, Lindegren et al. (2018) stress that a RUWE threshold should be set based on empirical evidence and not a theoretical distribution. For this, we can use the northern AGB star BX Cam as an example. Recent VLBI

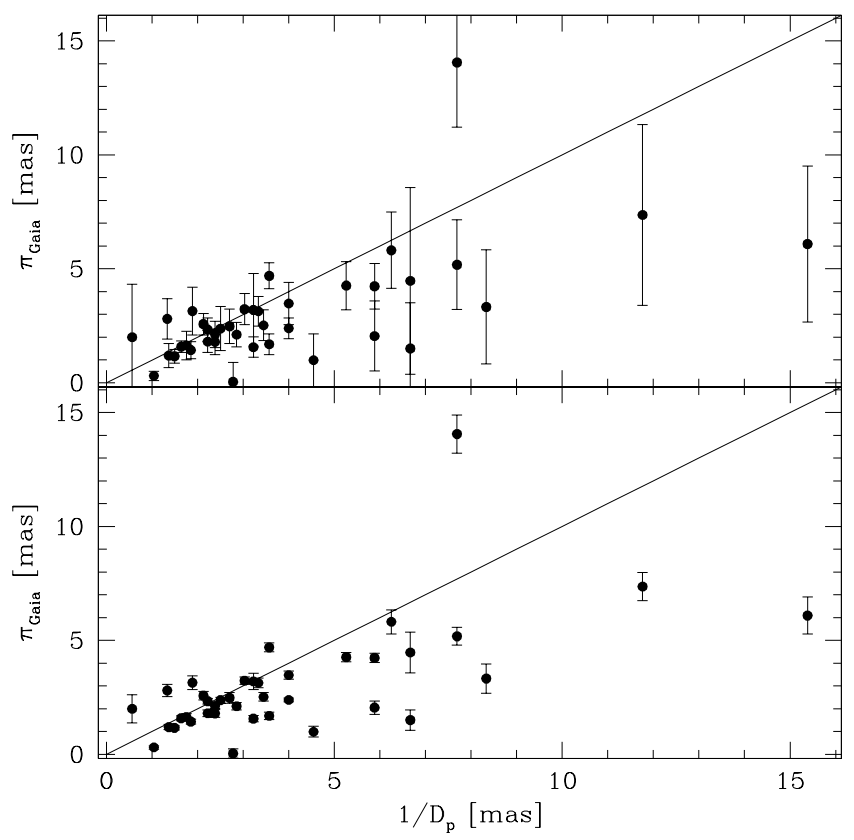

Fig. A.1. Gaia DR2 parallaxes for our sample compared to the distances used in the current mass-loss models. Bottom panel: the Gaia parallaxes are presented with their formal errors. Top panel: the astrometric_excess_noise value is added in quadrature to the formal errors following the empirical results from van Langevelde et al. (2018).

observations have revealed a parallax of $\pi_{\mathrm{VLBI}}=1.73 \pm 0.03$ mas (Matsuno et al. 2020). This value is significantly different from the Gaia value of $\pi_{\text {Gaia }}=4.13 \pm 0.25$ even when taking the astrometric_excess_noise of 0.67 mas into account. However, the RUWE of BX Cam is 1.04, which would have qualified as a good solution in most cases. In comparing the stellar luminosity, Matsuno et al. (2020) conclude that the larger VLBI distance is more reliable since the VLBI distance would imply a luminosity of $\sim 4950 L_{\odot}$, while the Gaia distance would result in a luminosity of only $\sim 870 L_{\odot}$. This example illustrates that even for AGB stars with an apparent low RUWE value, we should exercise caution when adopting the current Gaia DR2 parallaxes.

\footnotetext{
3 Lindegren et al. (2018), Gaia memo; GAIA-C3-TN-LU-LL-124-01.
} 
Appendix B: Imaging results

Table B.1. Imaging results.

\begin{tabular}{|c|c|c|c|c|c|c|c|c|c|c|c|c|}
\hline \multirow[b]{2}{*}{ Source } & \multicolumn{3}{|c|}{ Band 6} & \multicolumn{3}{|c|}{ Band 7} & \multicolumn{3}{|c|}{$\mathrm{CO}(2-1)$} & \multicolumn{3}{|c|}{$\mathrm{CO}(3-2)$} \\
\hline & $\begin{array}{c}\theta \\
{\left[{ }^{\prime \prime}\right]}\end{array}$ & $\begin{array}{l}\text { PA } \\
{\left[{ }^{\circ}\right]} \\
\end{array}$ & $\begin{array}{c}\mathrm{rms} \\
{\left[\frac{\mathrm{mJy}}{\text { beam }}\right]}\end{array}$ & $\begin{array}{c}\theta \\
{\left[{ }^{\prime \prime}\right]} \\
\end{array}$ & $\begin{array}{l}\mathrm{PA} \\
{\left[{ }^{\circ}\right]} \\
\end{array}$ & $\begin{array}{c}\mathrm{rms} \\
{\left[\frac{\mathrm{mJy}}{\text { beam }}\right]}\end{array}$ & $\begin{array}{c}F_{\text {peak }} \\
{[\mathrm{Jy}]}\end{array}$ & $\begin{array}{c}v_{\mathrm{c}} \\
{\left[\mathrm{km} \mathrm{s}^{-1}\right]}\end{array}$ & $\begin{array}{c}\Delta v \\
{\left[\mathrm{~km} \mathrm{~s}^{-1}\right]}\end{array}$ & $\begin{array}{c}F_{\text {peak }} \\
{[\mathrm{Jy}]}\end{array}$ & $\begin{array}{c}v_{\mathrm{c}} \\
{\left[\mathrm{km} \mathrm{s}^{-1}\right]}\end{array}$ & $\begin{array}{c}\Delta v \\
{\left[\mathrm{~km} \mathrm{~s}^{-1}\right]} \\
\end{array}$ \\
\hline \multicolumn{13}{|c|}{ M-type semi-regular and irregular stars: } \\
\hline $\mathrm{L}_{2}$ Pup & $6.5 \times 5.4$ & 67.0 & 52 & $3.9 \times 3.5$ & -70.1 & 103 & 19.8 & 33.3 & 7.5 & 47.2 & 32.7 & 8.0 \\
\hline W Hya & $6.6 \times 4.4$ & -89.1 & 51 & $4.5 \times 2.9$ & -89.9 & 340 & 36.0 & 41.0 & 17.0 & 68.0 & 41.0 & 17.5 \\
\hline T Mic & $6.3 \times 4.0$ & -78.2 & 76 & $4.9 \times 2.7$ & -79.1 & 168 & 17.5 & 25.3 & 12.5 & 20.0 & 25.0 & 13.0 \\
\hline Y Scl & $6.5 \times 4.3$ & 81.8 & 71 & $5.1 \times 2.7$ & 89.9 & 138 & 4.9 & 28.8 & 11.5 & 7.8 & 29.0 & 12.0 \\
\hline V1943 Sgr & $6.5 \times 4.2$ & -73.1 & 76 & $5.2 \times 2.7$ & -73.5 & 170 & 14.0 & -14.8 & 12.7 & 19.0 & -15.0 & 13.0 \\
\hline BK Vir & $7.6 \times 4.8$ & -89.0 & 112 & $4.9 \times 3.1$ & -67.2 & 144 & 12.9 & 16.8 & 11.5 & 25.8 & 17.0 & 11.0 \\
\hline V Tel & $6.5 \times 5.4$ & 74.3 & 52 & $4.4 \times 3.8$ & 84.9 & 155 & 11.9 & -32.5 & 16.0 & 21.3 & -32.8 & 15.5 \\
\hline SU Vel & $6.3 \times 4.5$ & -78.1 & 48 & $4.3 \times 3.2$ & 85.5 & 136 & 13.5 & 7.5 & 13.0 & 25.7 & 7.5 & 13.0 \\
\hline UY Cet & $6.2 \times 4.7$ & 88.1 & 72 & $5.2 \times 3.0$ & -69.5 & 142 & 9.5 & 4.7 & 15.3 & 18.1 & 4.8 & 15.5 \\
\hline SV Aqr & $6.4 \times 4.5$ & -82.9 & 40 & $4.3 \times 2.8$ & -89.9 & 158 & 4.0 & 5.6 & 18.8 & 5.8 & 6.8 & 18.5 \\
\hline SW Vir & $8.2 \times 4.6$ & -73.2 & 71 & $4.6 \times 3.3$ & 74.7 & 141 & 36.5 & -11.5 & 17.0 & 66.4 & -11.5 & 17.0 \\
\hline CW Cnc & $7.3 \times 4.6$ & -75.8 & 53 & $4.2 \times 3.4$ & 83.4 & 161 & 9.5 & 15.0 & 24.0 & 12.8 & 15.0 & 23.3 \\
\hline RT Vir & $8.8 \times 4.4$ & -70.8 & 72 & $4.7 \times 3.3$ & 68.2 & 145 & 16.9 & 17.0 & 18.0 & 31.0 & 17.3 & 17.5 \\
\hline $\mathrm{R} \mathrm{Crt}$ & $7.1 \times 4.4$ & 81.1 & 52 & $4.4 \times 2.8$ & -87.1 & 153 & 26.3 & 11.3 & 23.5 & 44.3 & 11.3 & 23.5 \\
\hline \multicolumn{13}{|c|}{ M-type Mira stars: } \\
\hline R Leo & $7.0 \times 4.7$ & -85.8 & 54 & $4.2 \times 3.2$ & 69.5 & 176 & 25.3 & -0.5 & 15.0 & 57.8 & -0.5 & 16.0 \\
\hline R Hya & $6.6 \times 4.4$ & -89.7 & 51 & $4.4 \times 2.8$ & -87.6 & 123 & 26.0 & -9.8 & 21.0 & 67.9 & -9.8 & 21.5 \\
\hline R Hor & $6.1 \times 5.4$ & 79.6 & 45 & $3.9 \times 2.8$ & -82.9 & 133 & 54.7 & 37.5 & 13.0 & 76.3 & 37.5 & 13.0 \\
\hline RR Aql & $6.2 \times 5.4$ & -73.5 & 50 & $4.9 \times 3.4$ & -68.3 & 97 & 19.0 & 27.8 & 16.5 & 27.1 & 27.8 & 16.5 \\
\hline IRC-10529 & $6.4 \times 5.2$ & -71.9 & 52 & $4.6 \times 3.4$ & -67.1 & 101 & 33.3 & -17.8 & 30.5 & 43.4 & -17.5 & 30.0 \\
\hline WX Psc & $7.4 \times 5.4$ & 31.1 & 89 & $4.4 \times 3.2$ & 70.5 & 129 & 28.6 & 9.0 & 39.0 & 38.9 & 9.5 & 39.0 \\
\hline IRC +10365 & $6.9 \times 5.6$ & 89.0 & 62 & $4.1 \times 3.4$ & -74.4 & 149 & 24.4 & -31.3 & 34.5 & 32.4 & -31.3 & 35.5 \\
\hline \multicolumn{13}{|c|}{ C-type semi-regular and irregular stars: } \\
\hline TW Oph & $10.3 \times 4.5$ & -84.8 & 72 & $4.5 \times 2.9$ & -89.3 & 94 & 6.3 & 28.4 & 17.3 & 9.5 & 28.5 & 17.0 \\
\hline NP Pup & $6.3 \times 5.2$ & 84.9 & 52 & $4.1 \times 3.5$ & -80.6 & 105 & 3.0 & 13.3 & 21.5 & 3.4 & 12.8 & 21.5 \\
\hline TW Hor & $6.1 \times 5.7$ & 81.0 & 41 & $4.0 \times 3.1$ & -77.2 & 112 & 13.9 & 1.3 & 14.5 & 24.1 & 1.1 & 13.8 \\
\hline $\mathrm{T}$ Ind & $6.0 \times 5.2$ & -84.9 & 47 & $5.0 \times 2.7$ & -72.7 & 168 & 5.3 & 15.8 & 12.5 & 8.6 & 15.8 & 11.5 \\
\hline RT Cap & $6.8 \times 3.9$ & -74.3 & 75 & $5.1 \times 2.6$ & -74.0 & 181 & 5.7 & -18.0 & 16.0 & 6.6 & -18.0 & 15.0 \\
\hline AQ Sgr & $6.4 \times 4.5$ & -82.6 & 47 & $6.0 \times 2.7$ & -79.7 & 179 & 8.2 & 21.5 & 22.0 & 9.7 & 21.5 & 23.0 \\
\hline U Hya & $6.9 \times 4.4$ & 86.7 & 51 & $4.3 \times 2.9$ & -86.6 & 144 & 43.3 & -31.3 & 14.5 & 63.1 & -31.3 & 14.5 \\
\hline W Ori & $6.3 \times 5.4$ & 81.3 & 49 & $4.0 \times 3.1$ & 85.5 & 146 & 11.3 & -1.5 & 23.0 & 18.0 & -1.5 & 23.0 \\
\hline V Aql & $7.7 \times 5.0$ & -68.7 & 62 & $4.3 \times 3.6$ & -79.6 & 123 & 12.8 & 53.5 & 19.0 & 22.9 & 53.8 & 19.5 \\
\hline Y Pav & $9.8 \times 5.0$ & 28.3 & 58 & $4.3 \times 3.8$ & 44.1 & 135 & 12.7 & -3.8 & 18.5 & 19.3 & -3.5 & 18.0 \\
\hline X Vel & $6.5 \times 4.4$ & -77.5 & 49 & $4.3 \times 3.2$ & 87.3 & 136 & 8.3 & -19.3 & 22.5 & 14.7 & -19.3 & 22.5 \\
\hline Y Hya & $7.5 \times 4.3$ & -74.8 & 54 & $4.6 \times 2.6$ & 89.3 & 146 & 10.7 & -8.5 & 19.0 & 15.0 & -8.5 & 18.0 \\
\hline SS Vir & $7.6 \times 4.5$ & -85.0 & 112 & $4.9 \times 3.0$ & -68.6 & 137 & 3.9 & 8.5 & 28.0 & 6.8 & 8.7 & 30.3 \\
\hline W CMa & $7.4 \times 4.2$ & -79.8 & 44 & $5.2 \times 2.6$ & 69.9 & 116 & 8.8 & -0.3 & 21.5 & 11.4 & -0.3 & 21.5 \\
\hline \multicolumn{13}{|c|}{ C-type Mira stars: } \\
\hline R Lep & $7.4 \times 3.8$ & -75.2 & 106 & $4.2 \times 2.7$ & 89.4 & 125 & 22.6 & 11.3 & 37.5 & 21.8 & 11.5 & 38.0 \\
\hline CZ Hya & $6.9 \times 4.3$ & -73.6 & 52 & $4.6 \times 2.7$ & 84.9 & 154 & 6.5 & 13.5 & 26.0 & 9.4 & 13.3 & 25.5 \\
\hline R For & $7.2 \times 4.0$ & -76.8 & 68 & $4.5 \times 3.1$ & 78.9 & 168 & 11.6 & -2.4 & 34.8 & 20.0 & -2.3 & 33.5 \\
\hline R Vol & $6.3 \times 6.0$ & -48.0 & 72 & $5.0 \times 3.6$ & -5.0 & 152 & 14.6 & -10.8 & 36.5 & 24.4 & -10.8 & 36.5 \\
\hline RV Aqr & $6.5 \times 5.4$ & -67.9 & 50 & $4.5 \times 3.0$ & -73.3 & 153 & 26.1 & 1.0 & 30.0 & 37.8 & 1.3 & 30.5 \\
\hline V688 Mon & $6.4 \times 4.6$ & -87.1 & 76 & $4.6 \times 2.9$ & -79.0 & 224 & 25.2 & 3.0 & 28.0 & 33.7 & 3.0 & 27.0 \\
\hline V1259 Ori & $6.2 \times 4.6$ & -79.5 & 77 & $5.1 \times 2.8$ & -71.1 & 229 & 28.9 & 43.0 & 31.0 & 48.0 & 43.3 & 28.5 \\
\hline
\end{tabular}

Notes. Columns 2-7 give the imaging results: full width at half-maximum beam-widths (major $\times$ minor axis), $\theta$, and position angle, PA, at the center frequency of ${ }^{12} \mathrm{CO}$ spectral windows, as well as the rms noise level in both band 6 and 7 , respectively, at a velocity resolution of $0.75 \mathrm{~km} \mathrm{~s}^{-1}$. Columns 8-13 give the CO line parameters: peak flux, center velocity, and total velocity width of the line profiles of both CO transitions presented in Figs. D.1-D.2. 


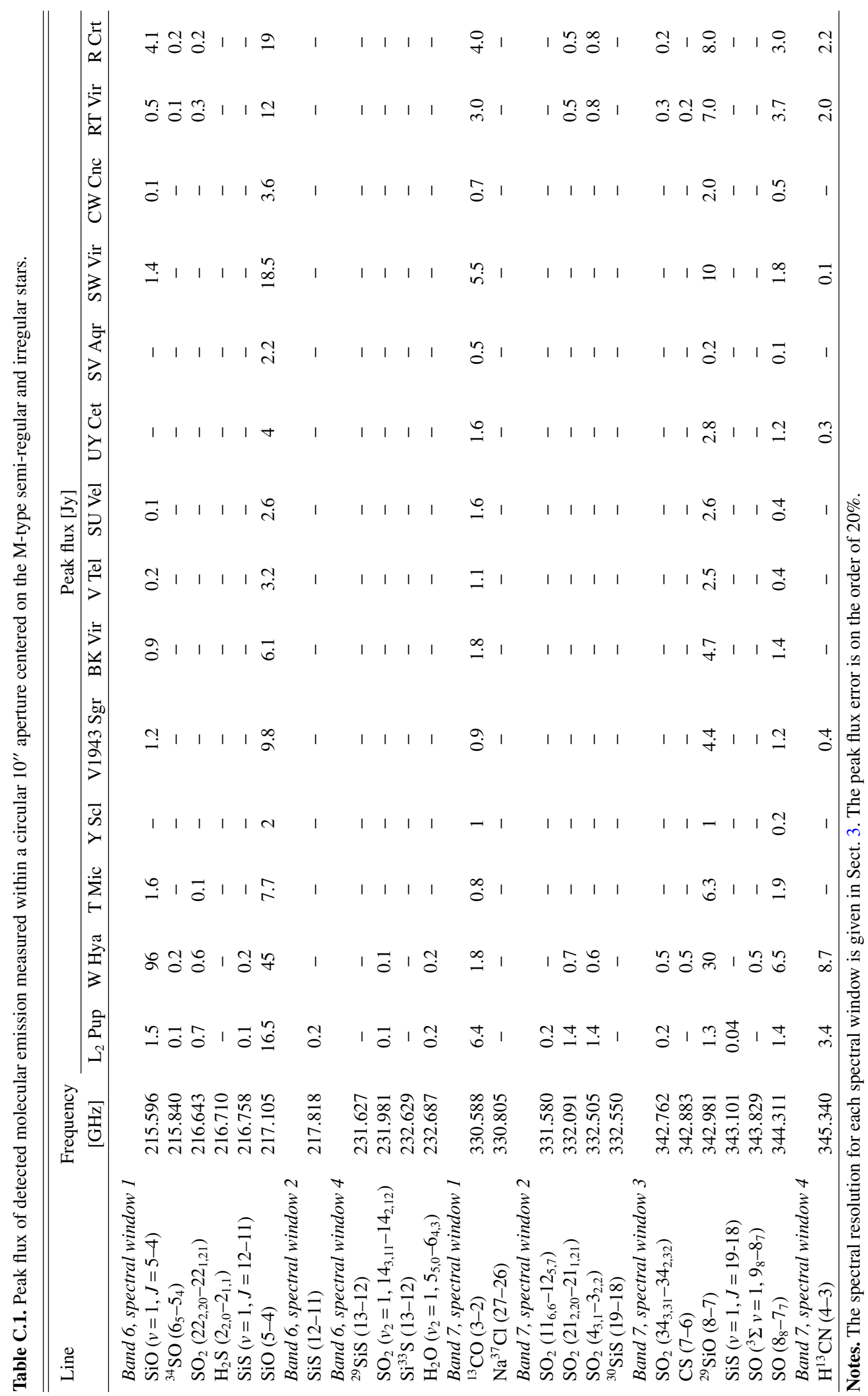


Table C.2. Peak flux of detected molecular emission measured within a circular 10" aperture centered on the M-type Mira stars.

\begin{tabular}{|c|c|c|c|c|c|c|c|c|}
\hline \multirow[t]{2}{*}{ Line } & \multirow{2}{*}{$\begin{array}{c}\text { Frequency } \\
{[\mathrm{GHz}]}\end{array}$} & \multicolumn{7}{|c|}{ Peak flux [Jy] } \\
\hline & & R Leo & R Hya & R Hor & RR Aql & IRC-10529 & WX Psc & IRC+10365 \\
\hline \multicolumn{9}{|l|}{ Band 6, spectral window 1} \\
\hline $\mathrm{SiO}(v=1, J=5-4)$ & 215.596 & 36 & 6.0 & 0.5 & 0.3 & 2.5 & 17 & 4.0 \\
\hline${ }^{34} \mathrm{SO}\left(6_{5}-5_{4}\right)$ & 215.840 & 0.1 & - & - & 0.1 & 0.1 & - & 0.1 \\
\hline $\mathrm{SO}_{2}\left(22_{2,20}-22_{1,21}\right)$ & 216.643 & 5.7 & - & - & 0.2 & - & - & - \\
\hline $\mathrm{H}_{2} \mathrm{~S}\left(2_{2,0}-2_{1,1}\right)$ & 216.710 & - & - & - & - & 0.3 & 0.2 & - \\
\hline $\operatorname{SiS}(v=1, J=12-11)$ & 216.758 & - & - & - & - & - & 0.1 & - \\
\hline $\mathrm{SiO}(5-4)$ & 217.105 & 36 & 22.4 & 6.7 & 4.2 & 5.7 & 9.6 & 12 \\
\hline \multicolumn{9}{|l|}{ Band 6, spectral window 2} \\
\hline $\operatorname{SiS}(12-11)$ & 217.818 & - & - & - & - & 1.8 & 2.9 & 0.9 \\
\hline \multicolumn{9}{|l|}{ Band 6, spectral window 4} \\
\hline${ }^{29} \mathrm{SiS}(13-12)$ & 231.627 & - & - & - & - & 0.2 & 0.4 & 0.1 \\
\hline $\mathrm{SO}_{2}\left(v_{2}=1,14_{3,11}-14_{2,12}\right)$ & 231.981 & - & - & - & - & - & - & - \\
\hline $\mathrm{Si}^{33} \mathrm{~S}(13-12)$ & 232.629 & - & - & - & - & 0.1 & - & - \\
\hline \multirow{2}{*}{\multicolumn{9}{|c|}{ Band 7 , spectral window 1}} \\
\hline & & & & & & & & \\
\hline${ }^{13} \mathrm{CO}(3-2)$ & 330.588 & 3.2 & 2.0 & 4.0 & 0.5 & 7.0 & 7.0 & 6.0 \\
\hline $\mathrm{Na}^{37} \mathrm{Cl}(27-26)$ & 330.805 & - & - & - & - & - & 0.1 & - \\
\hline \multicolumn{9}{|l|}{ Band 7 , spectral window 2} \\
\hline $\mathrm{SO}_{2}\left(11_{6,6}-12_{5,7}\right)$ & 331.580 & - & - & - & - & - & - & - \\
\hline $\mathrm{SO}_{2}\left(21_{2,20}-21_{1,21}\right)$ & 332.091 & 0.4 & - & - & 0.3 & - & - & - \\
\hline $\mathrm{SO}_{2}\left(4_{3,1}-3_{2,2}\right)$ & 332.505 & 0.4 & - & - & 1.9 & 1.2 & 1.2 & 0.8 \\
\hline${ }^{30} \mathrm{SiS}(19-18)$ & 332.550 & - & - & - & - & 0.3 & 0.5 & 0.2 \\
\hline \multicolumn{9}{|l|}{ Band 7, spectral window 3} \\
\hline $\mathrm{SO}_{2}\left(34_{3,31}-34_{2,32}\right)$ & 342.762 & 0.3 & - & - & - & - & - & - \\
\hline $\operatorname{CS}(7-6)$ & 342.883 & 0.7 & 0.2 & - & - & 0.7 & 1.4 & 1.0 \\
\hline${ }^{29} \mathrm{SiO}(8-7)$ & 342.981 & 21.5 & 12.5 & 7.0 & 2.0 & 1.3 & 2.7 & 3.1 \\
\hline $\operatorname{SiS}(v=1, J=19-18)$ & 343.101 & - & - & - & - & 0.3 & 0.4 & - \\
\hline $\mathrm{SO}\left({ }^{3} \Sigma v=1,9_{8}-8_{7}\right)$ & 343.829 & - & - & - & - & - & - & - \\
\hline $\mathrm{SO}\left(8_{8}-7_{7}\right)$ & 344.311 & 4 & 1.4 & 1.4 & 1.4 & 0.4 & 0.4 & 0.8 \\
\hline \multicolumn{9}{|l|}{ Band 7, spectral window 4} \\
\hline $\mathrm{H}^{13} \mathrm{CN}(4-3)$ & 345.340 & 7.7 & 0.9 & - & 1.4 & 0.6 & 1.7 & 1.6 \\
\hline
\end{tabular}

Notes. The spectral resolution for each spectral window is given in Sect. 3. The peak flux error is on the order of $20 \%$. 
S. Ramstedt et al.: DEATHSTAR: Nearby AGB stars with ALMA ACA

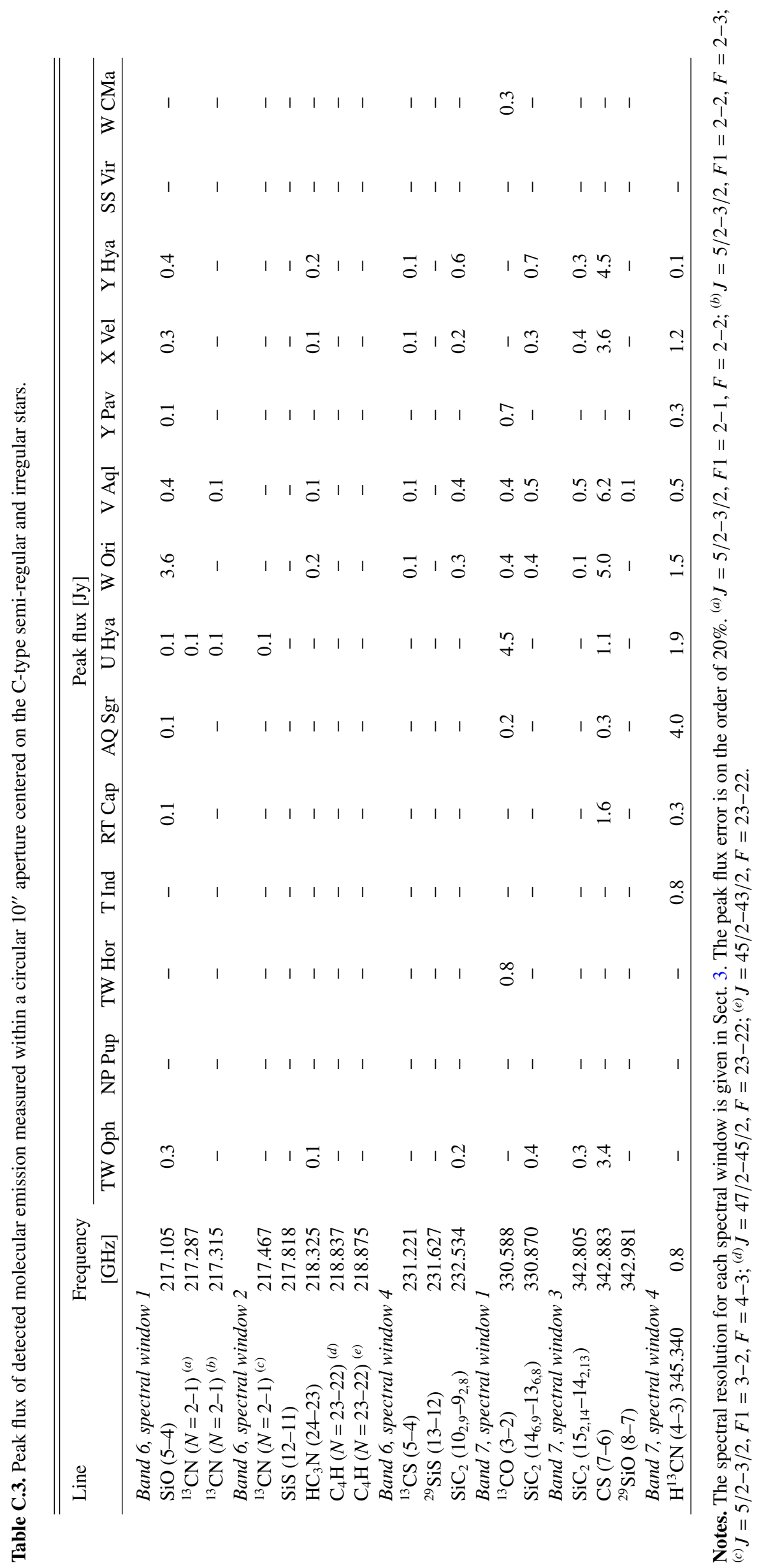


Table C.4. Peak flux of detected molecular emission measured within a circular 10" aperture centered on the C-type Mira stars.

\begin{tabular}{|c|c|c|c|c|c|c|c|c|}
\hline \multirow[t]{2}{*}{ Line } & \multirow{2}{*}{$\begin{array}{c}\text { Frequency } \\
{[\mathrm{GHz}]}\end{array}$} & \multicolumn{7}{|c|}{ Peak flux [Jy] } \\
\hline & & R Lep & CZ Hya & R For & R Vol & RV Aqr & V688 Mon & V1259 Ori \\
\hline \multicolumn{9}{|c|}{ Band 6, spectral window 1} \\
\hline $\mathrm{SiO}(5-4)$ & 217.105 & 1.9 & 0.3 & 2 & 1.9 & 3.2 & 1.0 & 0.8 \\
\hline${ }^{13} \mathrm{CN}(N=2-1){ }^{(a)}$ & 217.287 & 0.1 & - & 0.1 & - & 0.1 & - & - \\
\hline${ }^{13} \mathrm{CN}(N=2-1)^{(b)}$ & 217.315 & 0.1 & - & 0.2 & 0.1 & 0.2 & - & - \\
\hline \multicolumn{9}{|c|}{ Band 6, spectral window 2} \\
\hline${ }^{13} \mathrm{CN}(N=2-1){ }^{(c)}$ & 217.467 & 0.1 & - & 0.1 & 0.1 & 0.1 & - & - \\
\hline $\operatorname{SiS}(12-11)$ & 217.818 & - & - & 0.2 & - & 0.7 & 0.7 & 1.5 \\
\hline $\mathrm{HC}_{3} \mathrm{~N}(24-23)$ & 218.325 & 0.2 & - & 0.4 & 0.1 & 0.1 & 0.4 & 0.1 \\
\hline $\mathrm{C}_{4} \mathrm{H}(N=23-22)^{(d)}$ & 218.837 & - & - & - & - & - & 0.2 & 0.4 \\
\hline $\mathrm{C}_{4} \mathrm{H}(N=23-22)^{(e)}$ & 218.875 & - & - & - & - & - & 0.2 & 0.6 \\
\hline \multicolumn{9}{|c|}{ Band 6, spectral window 4} \\
\hline${ }^{13} \mathrm{CS}(5-4)$ & 231.221 & 0.2 & - & 0.2 & 0.2 & 0.3 & 0.1 & 0.2 \\
\hline${ }^{29} \mathrm{SiS}(13-12)$ & 231.627 & - & - & - & - & 0.1 & 0.1 & 0.1 \\
\hline $\mathrm{SiC}_{2}\left(10_{2,9}-9_{2,8}\right)$ & 232.534 & 0.1 & - & 0.3 & 0.2 & 0.3 & 0.4 & 0.4 \\
\hline \multicolumn{9}{|c|}{ Band 7, spectral window 1} \\
\hline${ }^{13} \mathrm{CO}(3-2)$ & 330.588 & 0.9 & 0.6 & 0.8 & 1.4 & 2.5 & 1.6 & - \\
\hline $\mathrm{SiC}_{2}\left(14_{6,9}-13_{6,8}\right)$ & 330.870 & - & - & 0.3 & 0.2 & 0.3 & 0.5 & - \\
\hline \multicolumn{9}{|c|}{ Band 7, spectral window 3} \\
\hline $\mathrm{SiC}_{2}\left(15_{2,14}-14_{2,13}\right)$ & 342.805 & - & - & 0.1 & - & - & - & - \\
\hline $\operatorname{CS}(7-6)$ & 342.883 & 5.6 & 0.5 & 6.8 & 6.5 & 11 & 5.6 & - \\
\hline${ }^{29} \mathrm{SiO}(8-7)$ & 342.981 & 0.1 & - & 0.5 & 0.3 & 0.5 & - & - \\
\hline \multicolumn{9}{|c|}{ Band 7, spectral window 4} \\
\hline $\mathrm{H}^{13} \mathrm{CN}(4-3)$ & 345.340 & 3.1 & 0.4 & 2.3 & 2.5 & 5 & 4 & - \\
\hline
\end{tabular}

Notes. The spectral resolution for each spectral window is given in Sect. 3 . The peak flux error is on the order of $20 \%$. ${ }^{(a)} J=5 / 2-3 / 2, F 1=2-2$, $F=2-3{ }^{(b)} J=5 / 2-3 / 2, F 1=2-1, F=2-2 ;^{(c)} J=5 / 2-3 / 2, F 1=3-2, F=4-3 ;{ }^{(d)} J=47 / 2-45 / 2, F=23-22 ;{ }^{(e)} J=45 / 2-43 / 2$, $F=23-22$. 


\section{Appendix D: Line profiles}
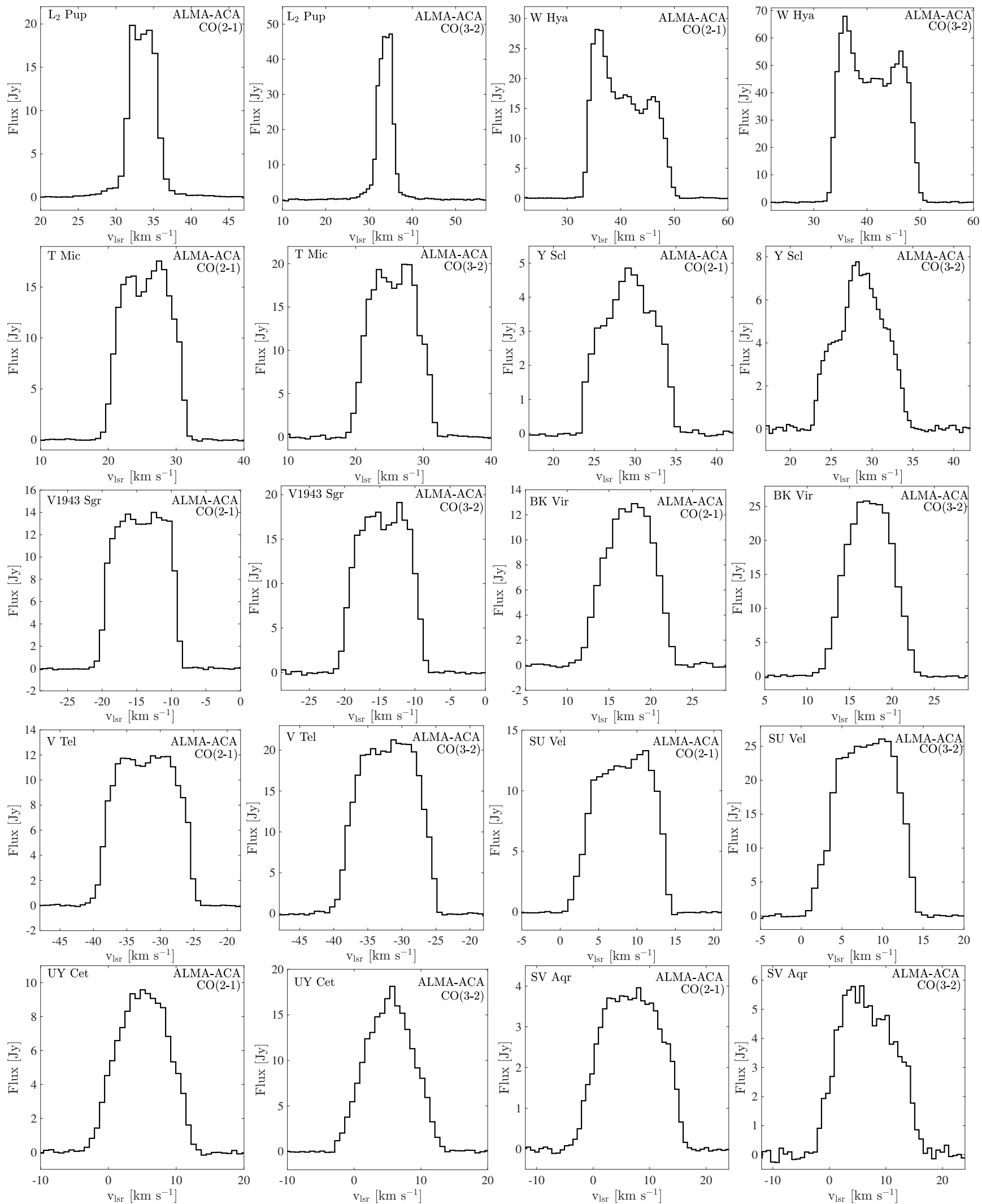

Fig. D.1. CO $J=2 \rightarrow 1$ and $3 \rightarrow 2$ line profiles measured toward the M-type AGB stars of the sample discussed in this paper. The source name is given in the upper left corner and the transition is in the upper right corner of each plot. 

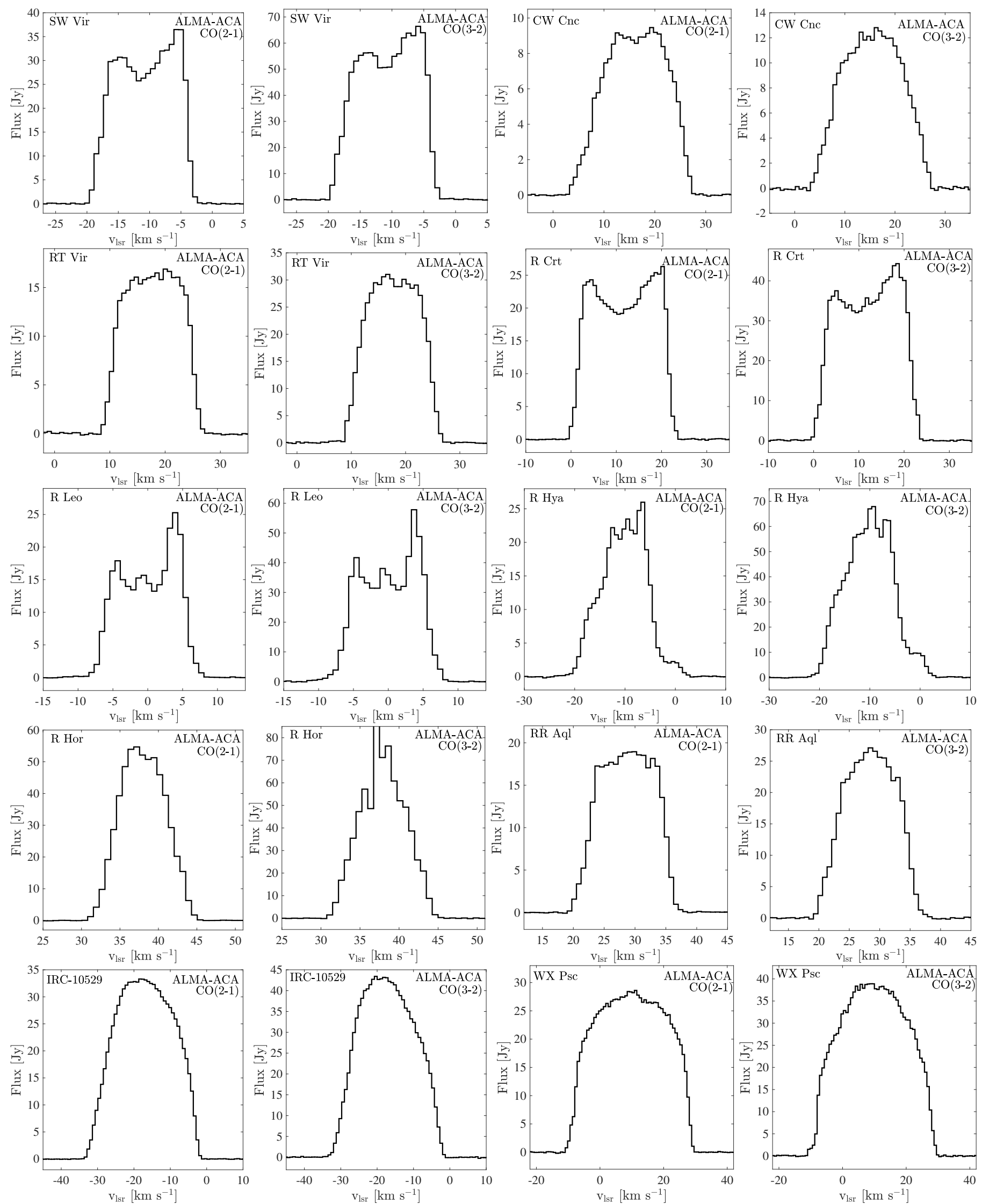

Fig. D.1. continued. 
S. Ramstedt et al.: DEATHSTAR: Nearby AGB stars with ALMA ACA
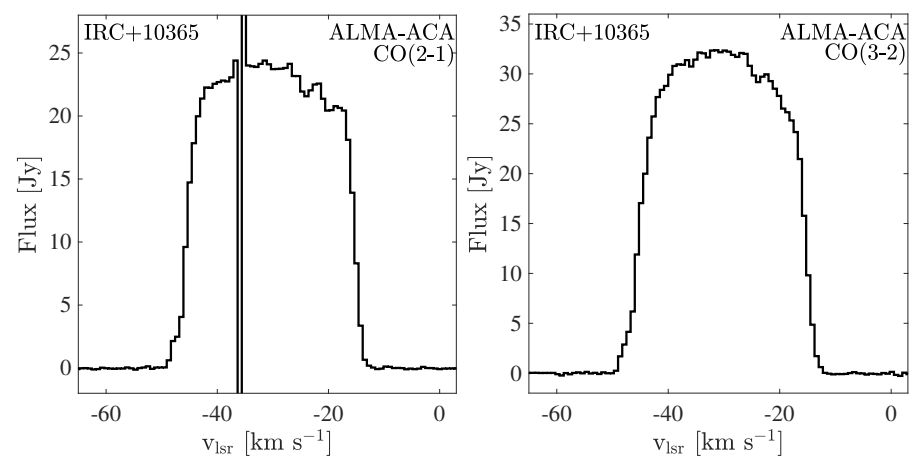

Fig. D.1. continued. 
A\&A 640, A133 (2020)
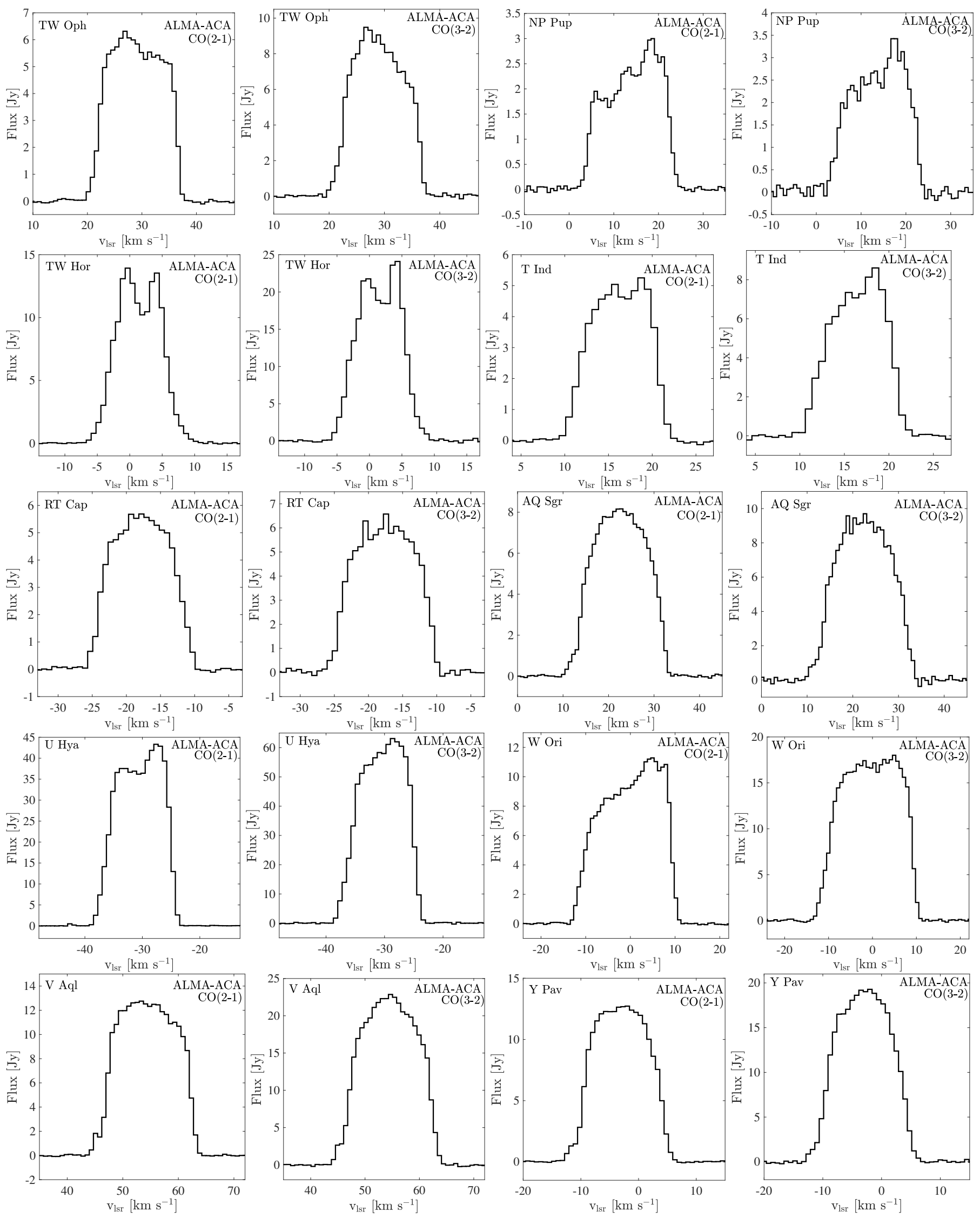

Fig. D.2. CO $J=2 \rightarrow 1$ and $3 \rightarrow 2$ line profiles measured toward the C-type AGB stars of the sample discussed in this paper. The source name is given in the upper left corner and the transition is in the upper right corner of each plot. 
S. Ramstedt et al.: DEATHSTAR: Nearby AGB stars with ALMA ACA
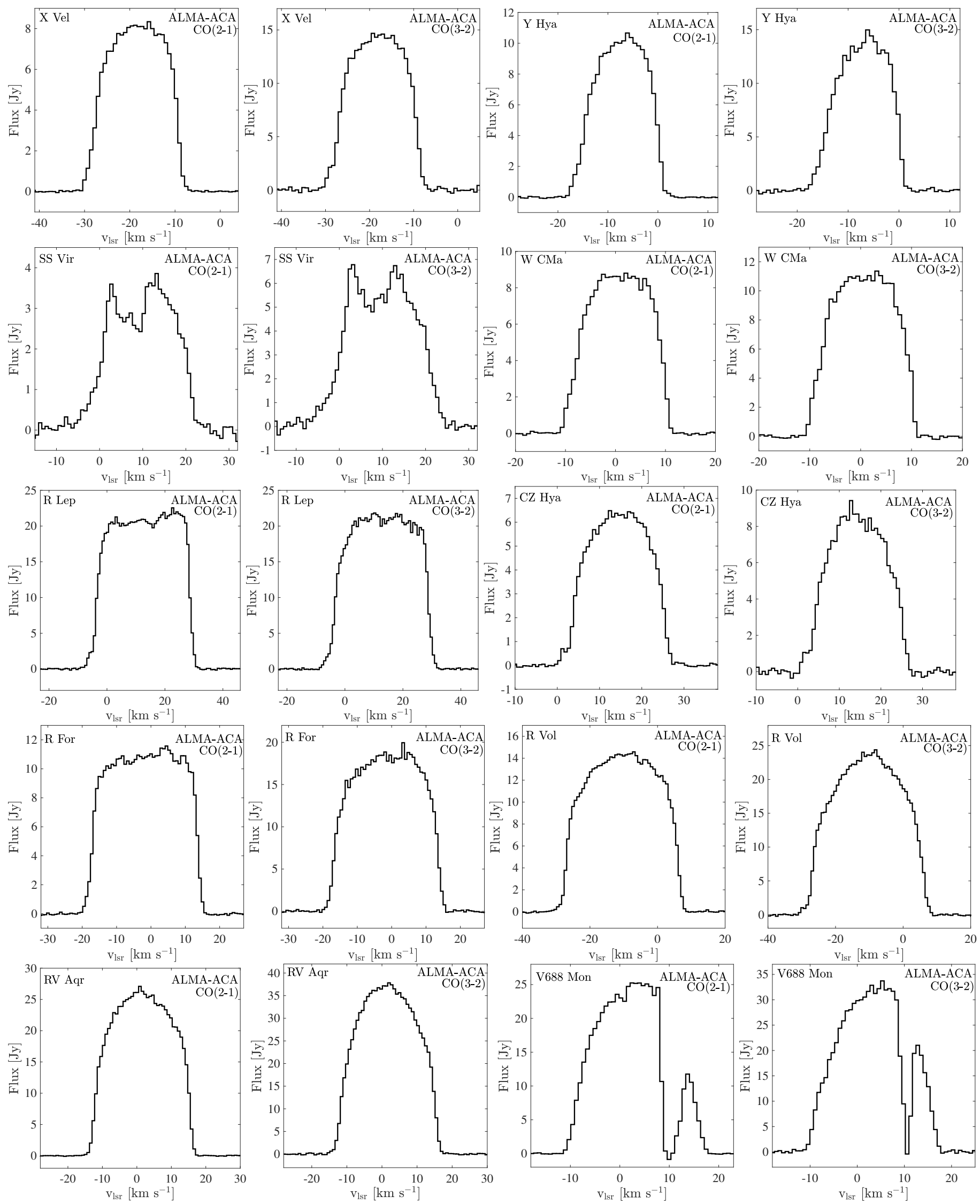

Fig. D.2. continued. 
A\&A 640, A133 (2020)
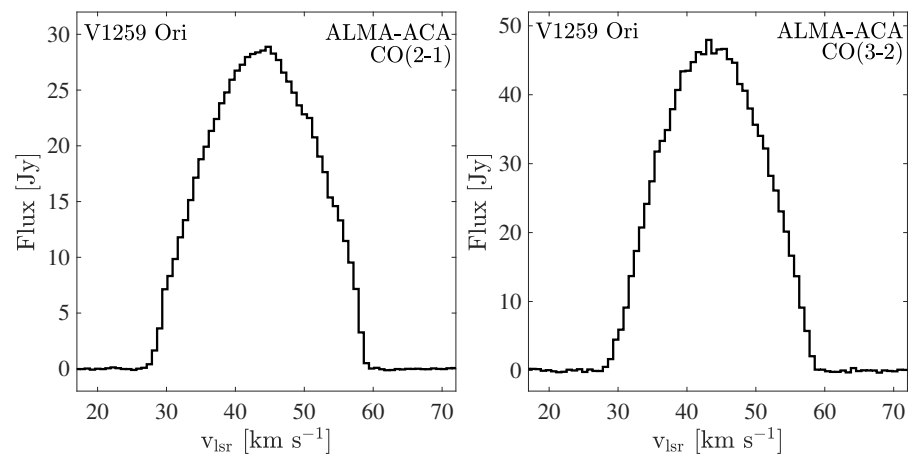

Fig. D.2. continued. 


\section{Appendix E: Results from fitting to Gaussian emission distributions}
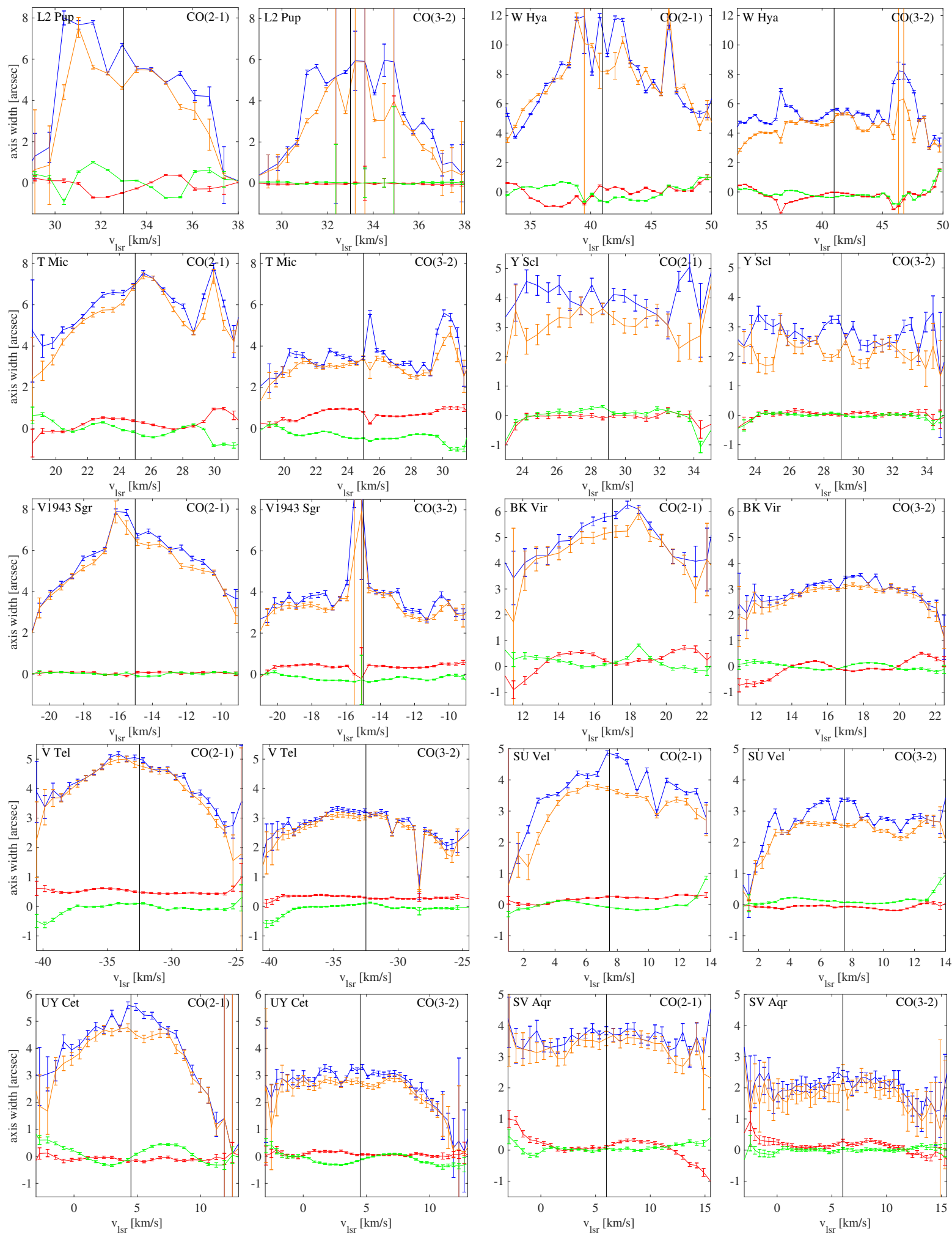

Fig. E.1. Results from the visibility fitting to the data measured toward the M-type AGB stars of the sample discussed in this paper. The source name is given in the upper left corner and the transition is in the upper right corner of each plot. The upper blue and orange lines show the major and minor axis of the best-fit Gaussian in each channel, respectively. The lower red and green lines show the RA and Dec offset relative to the center position, respectively. 
A\&A 640, A133 (2020)
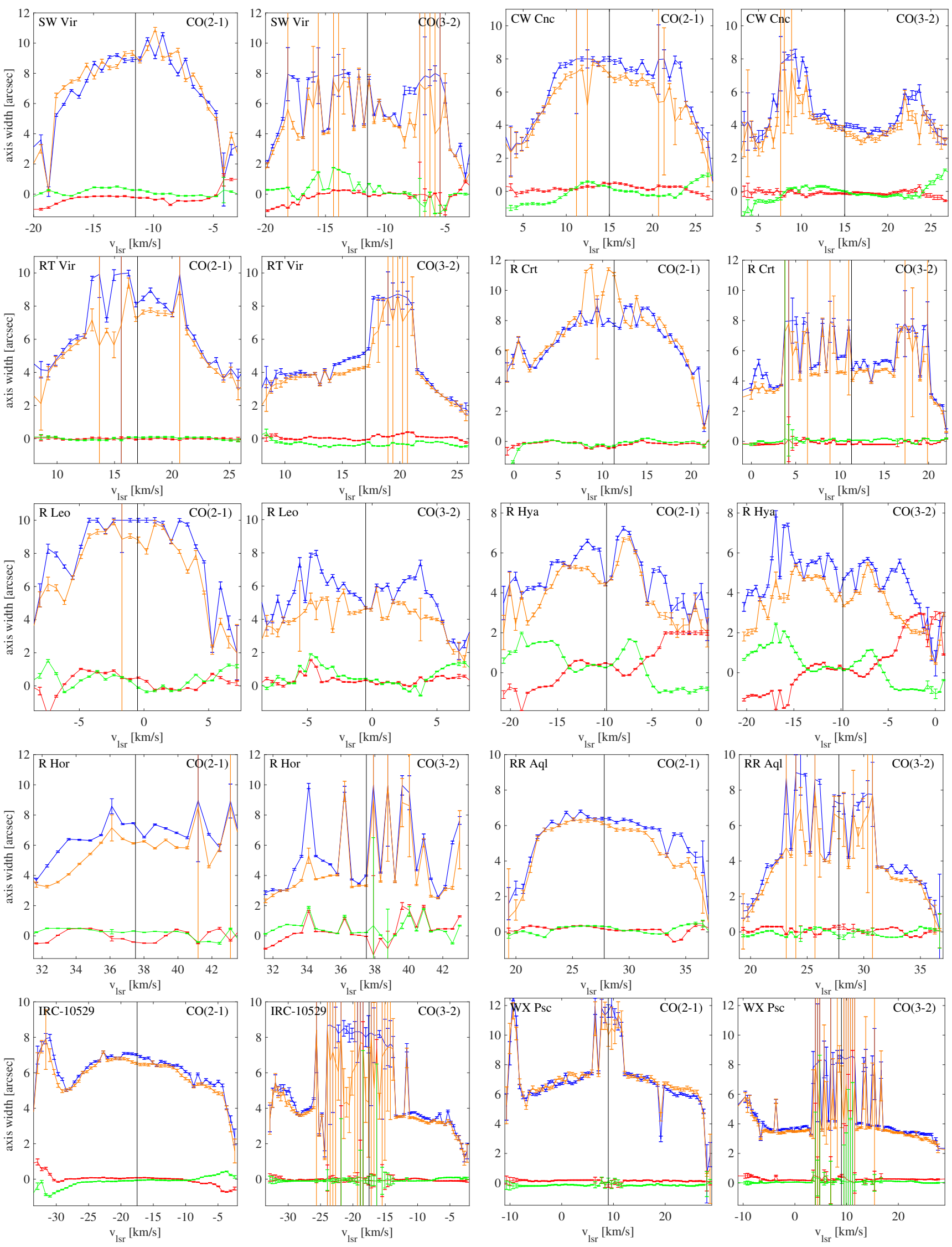

Fig. E.1. continued. 
S. Ramstedt et al.: DEATHSTAR: Nearby AGB stars with ALMA ACA
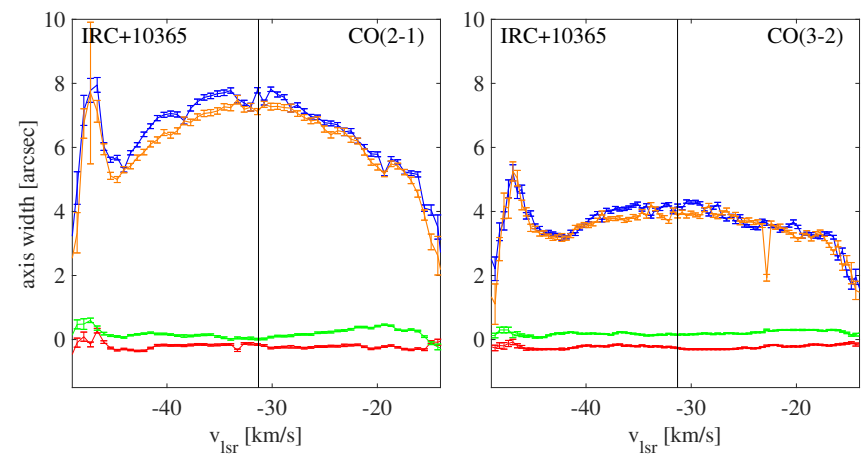

Fig. E.1. continued. 

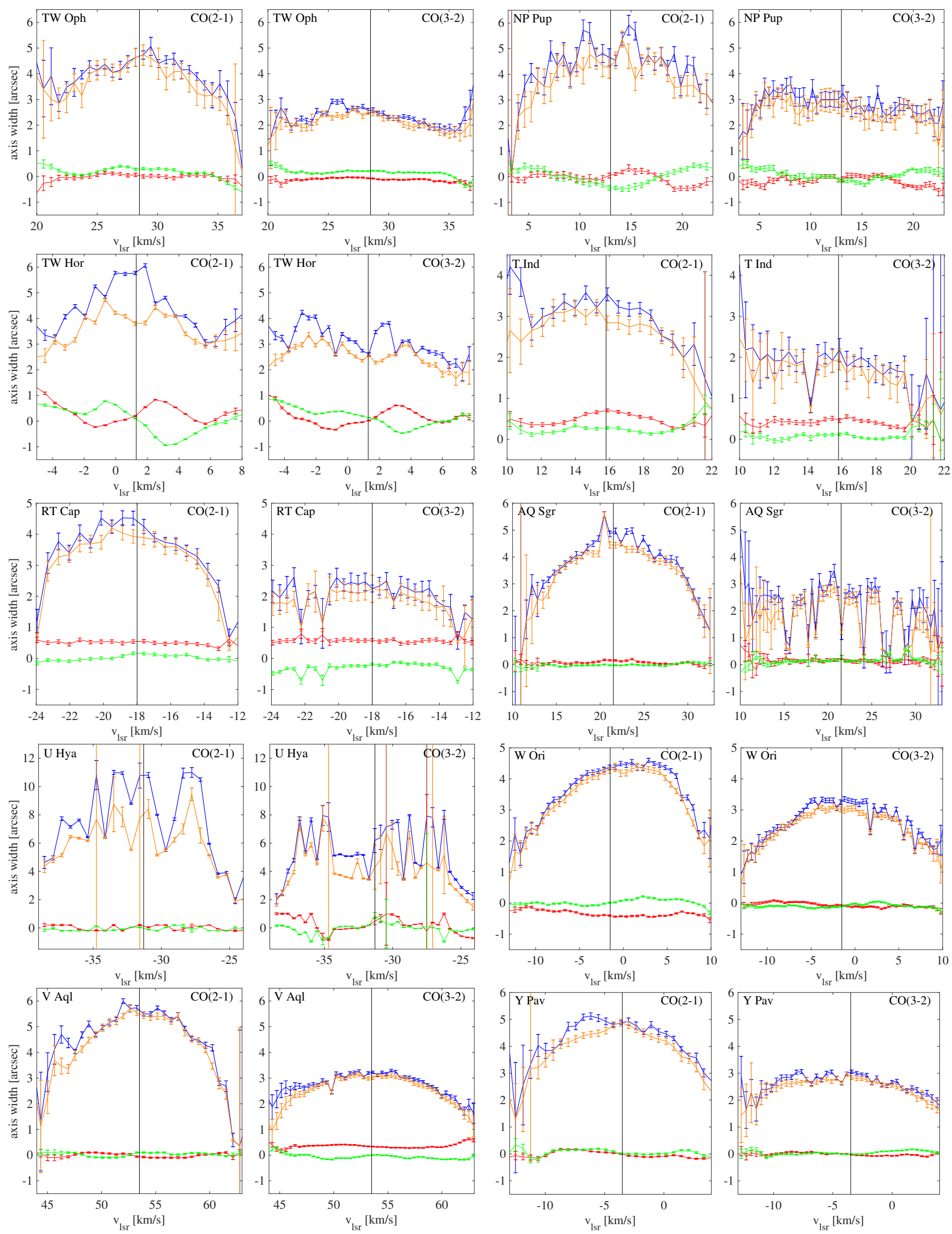

Fig. E.2. Results from the visibility fitting to the data measured toward the C-type AGB stars of the sample discussed in this paper. The source name is given in the upper left corner and the transition is in the upper right corner of each plot. The upper blue and orange lines show the major and minor axis of the best-fit Gaussian in each channel, respectively. The lower red and green lines show the RA and Dec offset relative to the center position, respectively. 
S. Ramstedt et al.: DEATHSTAR: Nearby AGB stars with ALMA ACA
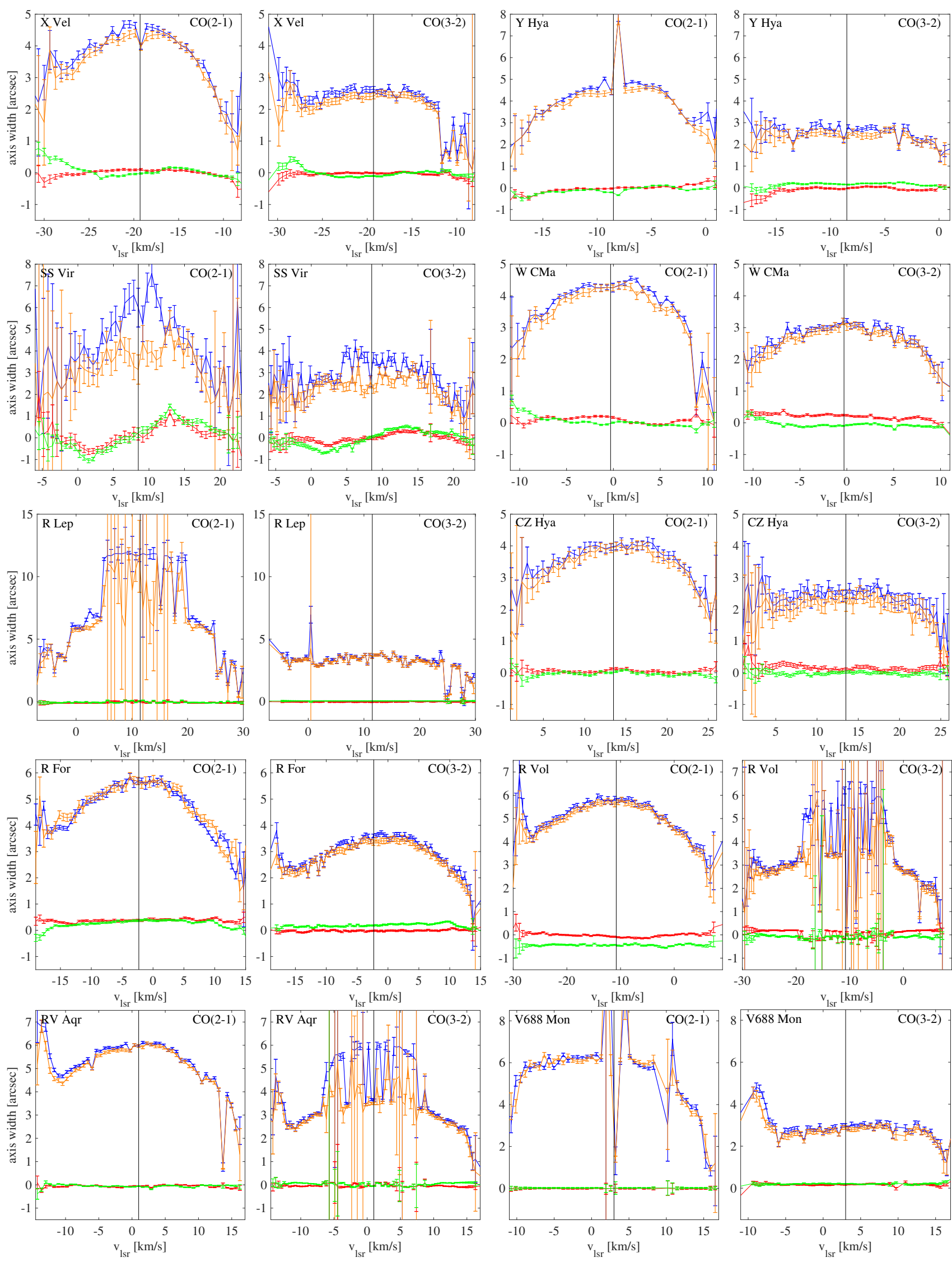

Fig. E.2. continued. 
A\&A 640, A133 (2020)
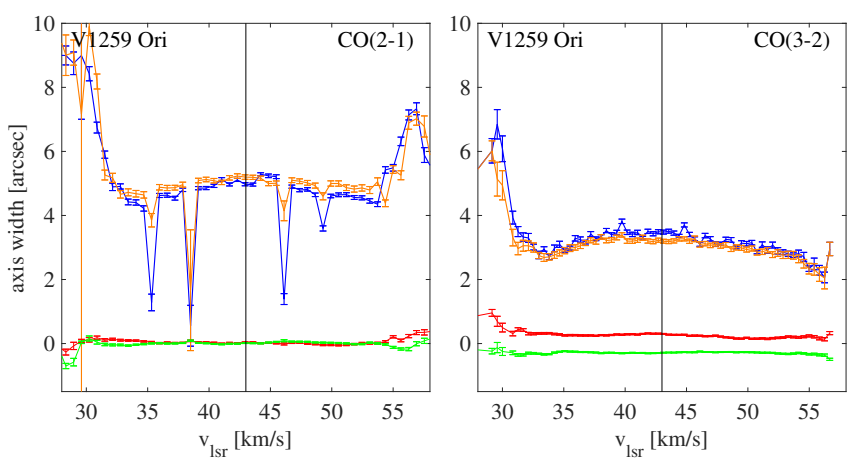

Fig. E.2. continued. 